\title{
New liverwort (Marchantiophyta) records for the Fiji Islands
}

\author{
Tamás Pócs ${ }^{1}$, Andrea Sass-Gyarmati ${ }^{1}$, Alifereti Naikatini², Marika \\ Tuiwawa², John Braggins ${ }^{3}$, Sarolta Pócs', \\ and Matt von Konrat ${ }^{4}$
}

\author{
${ }^{1}$ Botany Department, Eszterházy College, Eger. Pf. 43, H-3301, Hungary \\ ${ }^{2}$ Institute of Applied Science, University of the South Pacific, Private Bag, Suva, Fiji \\ ${ }^{3}$ Auckland War Memorial Museum, Auckland, New Zealand \\ ${ }^{4}$ Botany Department, The Field Museum, Chicago, Illinois 60605-2496, U.S.A.
}

\begin{abstract}
Forty-eight species of liverworts (Marchantiophyta) are reported as new to the Fiji Islands. These records increase the number of Fijian liverworts by over 20\%. Extensions of range within Fiji are reported for an additional 39 taxa already known to occur within the Fijian archipelago. These records indicate that the Fijian flora is understudied and many localities in Fiji, including entire islands, remain under-collected for bryophytes. Metalejeunea crassitexta (J.B.Jack et Steph.) Pócs is proposed as a new combination, and Lejeunea microstipula Steph. is reduced to a synonym of Lejeunea exilis (Reinw., Blume et Nees) Grolle.
\end{abstract}

\section{Introduction}

The Republic of Fiji consists of over 300 islands of varying sizes with an aggregate land area of about $18,300 \mathrm{~km}^{2}$ as well as many smaller islets and off-shore rocks (DoE 1997). Many localities in Fiji, including entire islands, remain under-collected for many plant groups (DoE 1997). Von Konrat et al. (2011, this issue) provides an overview of the current state of floristic knowledge for Fijian liverworts. Historically, very few publications have focused solely on collections from Fiji. However, in the last decade, there has been an increasing interest in the Fijian liverwort flora, as exemplified by Pócs (2008a, b), Pócs \& Eggers (2007) and field programs. This paper reports on new records, mostly collected by the first author with his wife, Sarolta Pócs, in 2003, as well as those on a joint expedition in 2008 organised by Matt von Konrat (F). The records are divided into two parts: a) new records for the Fiji Islands, represented by 48 taxa; and b) records for 39 taxa that are island range extensions. These reports cover the highlands of Taveuni, Viti Levu and Kadavu. 


\section{Methodology and materials}

If not otherwise stated, the specimens were identified by the senior author. All specimens are deposited in EGR, with duplicates in F and SUVA. To reduce the length of the paper, and to avoid unnecessary duplication of detailed locality data, abbreviated specimen data is provided in the 'Specimens examined' section and full locality data can be cross referenced by the collection number in Appendix 1. For example, an abbreviated collection appears as follows in the text: Taveuni Island: T. Pócs 08013/H. In Appendix 1, this corresponds to: 08013 - TAVEUNI Island, NW slope of DES VOEUX (DEVO) peak, below PWD Weather Station, at 900-1100 m alt. S 16 $50^{\prime} 18-28^{\prime \prime}, \mathrm{W} 179^{\circ}$ 57'57-58". Montane mossy forest. 11 Apr. 2008. The alphabetical letters that typically follow the collection numbers of the first author refer to multiple collections at the same locality. For many taxa the distribution has been extracted from the Early Land Plant Today (ELPT) databases of Söderström and Hagborg to supplement cited literature. The distribution and mapping of ELPT is described by von Konrat et al. (2010). Type data also are provided. Sources include the ELPT database and the online version of Index Hepaticarum (see: http: //www.ville-ge.ch/musinfo/bd/cjb/hepatic/index.php). Type data is typically presented with the type country, followed by type information directly derived from the protologue. Where we have not been able to cite data directly from the published protologue, we defer to Index Hepaticarum (IH) online, which we cite as the source. In many cases the type data is cited directly from a cited reference.

\section{Nomenclatural novelties}

The following two nomenclatural novelties are made. The rationale is described in detail under the respective species in the main body of the list.

Lejeunea exilis (Reinw., Blume et Nees) Grolle, Journal of the Hattori Botanical Laboratory 46: 353 (1979)

Basionym: Jungermannia exilis Reinw., Blume et Nees, Nova Acta Physico-Medica Academiae Caesareae Leopoldino-Carolinae Naturae Curiosorum Exhibentia Ephemerides sive Observationes Historias et Experimenta 12: 227 (1824) [1825]

Synonym: Lejeunea microstipula Steph. Hedwigia 29: 89, 139, XVI (1890) "MicroLejeunea" syn. nov.

Metalejeunea crassitexta (J.B.Jack et Steph.) Pócs, comb. nov.

Basionym: Microlejeunea crassitexta J.B.Jack et Steph., Botanisches Centralblatt 60: 106 (1894)

\section{New records for the Fiji Islands}

The following 48 taxa are reported new to the islands of Fiji.

1. Bazzania falcifolia (Steph.) H.A.Mill., Phytologia 47: 320 (1981)

Basionym: Mastigobryum falcifolium Steph., Denkschriften der Kaiserlichen Akademie der Wissenschaften, Wien. Mathematisch-Naturwissenschaftliche Klasse 88: 33 (1911) 
Type citation: Samoa, “Insel Upolu. In Urwäldern bei Tiavi, auf Bäumen. Nr. 5370” leg. Rechinger. Source: IH online.

Illustration: Stephani (1985: 6879).

Specimens examined: Taveuni Island: T. Pócs 08013/H.

Bazzania falcifolia has previously been reported for New Caledonia and Samoa (Miller et al. 1983).

2. Bazzania manillana (Gottsche ex Steph.) S.Hatt., Botanical Magazine, Tokyo 64: 113 (1951)

Basionym: Mastigobryum manillanum Gottsche ex Steph., Hedwigia 25: 204 (1886)

Type citation: Philippines, "Manila, leg. Gaudichaud (Holotype: hb. Gottsche)".

Illustration: Stephani (1985: 007142).

Specimens examined: Taveuni Island: T. Pócs 08013/D.

Bazzania manillana has previously been reported for Peninsular Malaysia, Philippines, Indonesia (Java), New Guinea, and Samoa (cf. ELPT database).

3. Cheilolejeunea lindenbergii (Gottsche) Mizut., Journal of the Hattori Botanical Laboratory 33: 226 (1970)

Basionym: Lejeunea lindenbergii Gottsche, Synopsis Hepaticarum 3: 336 (1845)

Type citation: Bangladesh, "prope Sylhet, Indiae orientalis (Wallich in Hb. L[ehmann])" - Sri Lanka "in Insula Ceylon... (Hb. H[oo]k[er]) n. 51 ex annotationibus Lindenbergii. Hb. L[ehmann] et N[ees])”.

Illustration: Mizutani (1967: 174, fig.III as Cheilolejeunea luerssenii).

Specimens examined: Taveuni Island: S. \& T. Pócs 03299/P.

Cheilolejeunea lindenbergii was previously thought to be endemic to the Malesian region (Piippo 1994).

4. Cheilolejeunea occlusa (Herzog) T.Kodama et N.Kitag., Bulletin of the Osaka Museum of Natural History. Osaka. 28: 40 (1974).

Basionym: Strepsilejeunea occlusa Herz., Transactions of the British Bryological Society 1: 320 (1949)

Type citation: Malaysia (Sarawak), "Dulit Ridge, c. 1230 m, rambling on Chiloscyphus aselliformis var. neesii, on tree trunks in open 'Mossy Forest”, no. 2200 p.p.”.

Illustration: Mizutani (1980: 324, fig. 3).

Specimen examined: Viti Levu Island: S. \& T. Pócs 03274/AT.

Cheilolejeuna occlusa is also known from Indonesia (Sumatra and Borneo) (Mizutani 1980). 


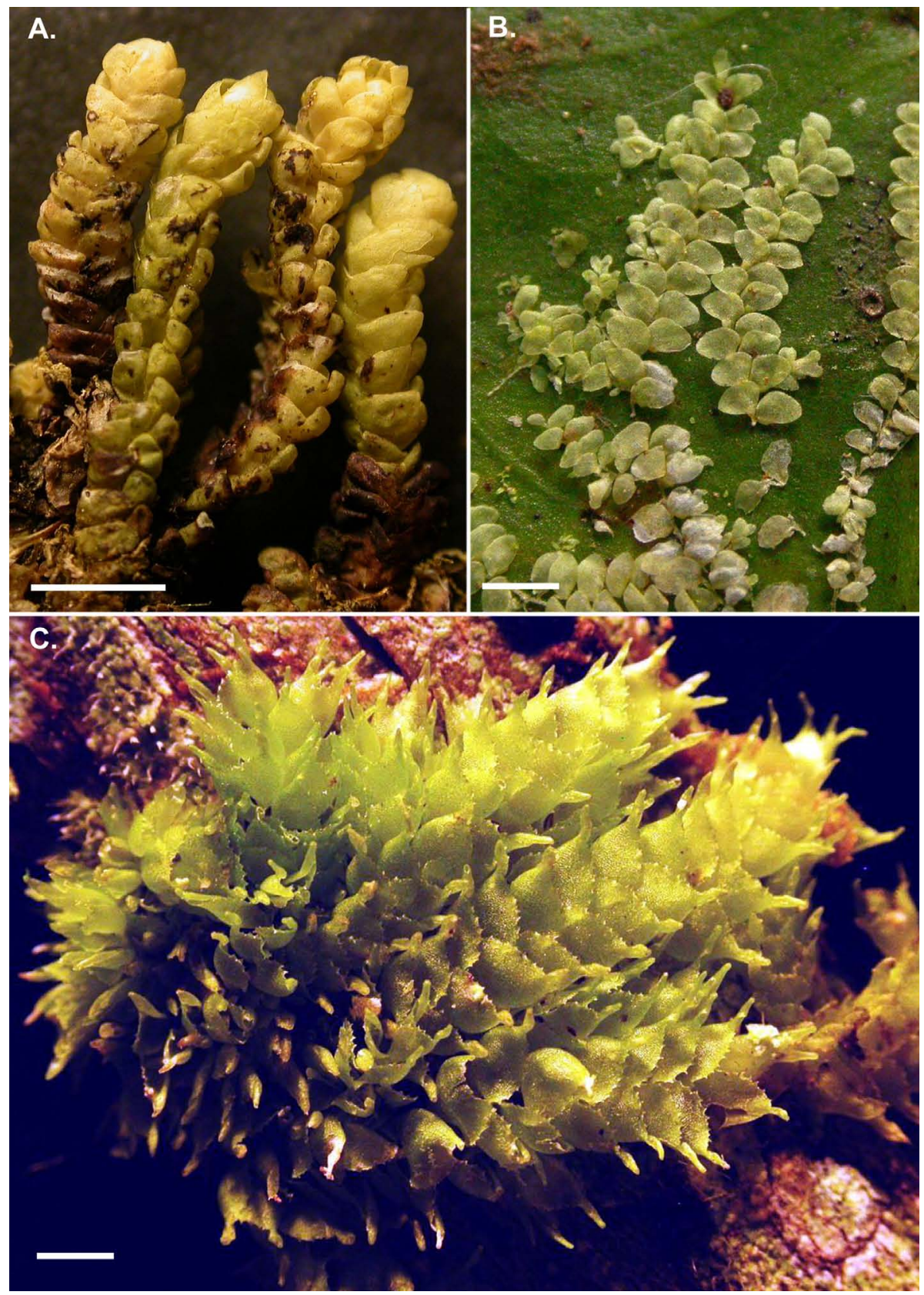

Fig. 1. A, Pleurozia conchifolia, habit (S. \& T. Pócs 03279/N), scale bar $2.5 \mathrm{~mm}$; B, Cololejeunea aequabilis, epiphyllous habit (S. \& T. Pócs 03280/CP), scale bar $1 \mathrm{~mm}$; C, Colura brevistyla, habit on bark (T. Pócs 08036/V), scale bar $1 \mathrm{~mm}$. 
5. Cololejeunea aequabilis (Sande Lac.) Schiffn., Conspectus Hepaticarum Archipelagi Indici: 242 (1898)

Fig. 1b

Basionym: Lejeunea aequabilis Sande Lac., Annales Museum Botanicum LugdunoBatavi 1: 310 (1864)

Type citation: Indonesia. Java, "prov. Bantam, in foliis Anonaceae", leg. Blume.

Illustration: Zhu et al. (1999: 294, fig.1 as Cololejeunea yulensis).

Specimens examined: Taveuni Island: S. \& T. Pócs 03280/BK, 03280/BP; Viti Levu Island: $S$. \& T. Pócs 03271/AB, 08037/E; A.J. Fife 08037/G, Q.

Cololejeunea aequabilis appears to be a Southeast Asian and Oceanian species, occurring in China, Indonesia, Japan, Vietnam, and Samoa (Zhu 2005).

6. Cololejeunea amphibola B.M.Thiers, Beihefte zur Nova Hedwigia 90: 130 (1988)

Type citation: Australia. “Queensland. Cook Dist.: Millaa Millaa Falls National Park, $900 \mathrm{~m}, 17^{\circ} 31^{\prime} \mathrm{S}, 145^{\circ} 38^{\prime} \mathrm{E}$. Thiers \& Halling 2210b, Jul 5, 1984 (Holotype: AD, isotype: NY)".

Illustration: Thiers (1998: 131, pl.1).

Specimens examined: Taveuni Island: S. \& T. Pócs 03279/CC, 03280/CL, 03289/BK pp; Viti Levu Island: S. \& T. Pócs 03274/BH, BY.

Cololejeunea amphibola has previously been reported for Borneo, Sulawesi, New Guinea, and Australia (Queensland) (Eggers et al. 1998).

7. Cololejeunea angustiflora (Steph.) Mizut., Journal of the Hattori Botanical Laboratory 28: 113 (1965)

Basionym: Leptocolea angustiflora Steph., Species Hepaticarum 5: 848 (1915)

Type citation: New Guinea. "Nova Guinea" No detailed collection data.

Illustration: Mizutani (1965: 114, fig. IV); Zhu \& So (2002: 432, figs 1-23).

Specimens examined: Taveuni Island: S. \& T. Pócs 0279/AV, 0280/CQ.

Cololejeunea angustiflora has previously been reported from China (Taiwan), Philippines, Indonesia, Malaysia, Papua New Guinea, and New Caledonia (Zhu \& So 2002).

8. Cololejeunea cardiocarpa (Mont.) A.Evans, Memoirs of the Torrey Botanical Club 8: 172 (1902)

Basionym: Lejeunea cardiocarpa Mont., de la Sagra, Histoire Physique, Politique et Naturelle de l'île de Cuba, Botanique, Plantes Cellulaires: 476 (1842)

Type citation: Cuba. "Ad folia Calophylli Calabae repentem in Cuba insula legerunt hanc speciem clarr. Ramon de la Sagra et Auber" (Isotype: PC!).

Illustration: Schuster (1980: 1279, fig. 765); Asthana \& Srivastava (2003: 100, pl. 16). Specimens examined: Viti Levu Island: S. \& T. Pócs 03260/N.

Cololejeunea cardiocarpa is a widespread species known from the Neotropics with 
outliers in the south-eastern USA, Africa, and records from New Caledonia, tropical Australia, and New Zealand (Braggins 1999) as well as from Tonga and Tahiti Is. (Hürlimann 1987).

9. Cololejeunea cocoscola Tixier, Cryptogamie: Bryologie, Lichénologie 14: 353 (1993)

Type citation: Tonga. "Ouest de Neiafu (Vava'u), 50 m., Hürlimann T873a (Holotype: hb. Hürlimann)".

Illustration: Tixier (1993: 354, fig. 1).

Specimens examined: Kadavu Island: S. \& T. Pócs 03302/C, 03303/J, 03303/AN; Viti Levu Island: S. \& T. Pócs 03255/B, 03267/C; Taveuni Island: S. \& T. Pócs 03283/O, 03284/C,03285/BB, 03287/J, T Pócs 08015/C.

Cololejeunea cocoscola had previously been reported only from Tonga (Miller et al. 1983).

10. Cololejeunea diaphana A.Evans, Bulletin of the Torrey Botanical Club 32: 184 (1905)

Type citation: U.S.A., Florida, "in hammocks near the homestead trail, between Cutler and Camp Longview, leg. Small \& Carter 1365 p. p., 1370 p. p.”.

Illustration: Evans (1905: 184, pl. 5); Schuster (1980: 1295, fig. 765; 1980: 1299, fig. 769 as Aphanolejeunea diaphana).

Specimens examined: Taveuni Island: S. \& T. Pócs 03280/BJ, 03288/CY, 03289/CV.

Cololejeunea diaphana is new to the South Pacific. Pócs \& Bernecker (2009) recognised C. diaphana as a Pantropical taxon distributed in tropical America, Africa and Asia.

11. Cololejeunea equialbi Tixier, Annales de la Faculté des Sciences, Université de Phnom Penh 3: 178 (1970)

Type citation: Vietnam. Thua Thien, Bach Ma, alt. 1400 m, Tixier 2309, 1962; Holotype, PC (Tixier 1985).

Illustration: Tixier (1985: 304, fig. 63); Zhu \& So (2001: 287, fig. 108).

Specimens examined: Kadavu Island: S. \& T. Pócs 03309/BD; Taveuni Island: S. \& T. Pócs 03290/ $B D$.

Cololejeunea equialbi is a Southeast Asian species (Piippo 1994), with records from China (Gansu, Shanxi, Yunnan, Hainan), Japan (Nansei-Shoto (Ryu-Kyu Is)), Vietnam, Philippines, Indonesia (Sulawesi, Moluccas), and New Guinea (c.f. ELPT database).

12. Cololejeunea falcata (Horik.) Benedix, Feddes Repertorium Specierum Novarum Regni Vegetabilis. Beiheft 134: 29 (1953)

Basionym: Physocolea falcata Horik., Journal of Science of Hiroshima University, Series B, Division 2 (Botany) 1: 22. f. 6. (1931)

Type citation: Japan. Bonin Islands [= Ogasawara Gunto] "Bonin: Insl. Chichijima (Y. Horikawa no. 1962a-type, July 1930)” (Holotype: HIRO). Source: IH online. 
Illustration: Tixier (1979: 738, fig. 9); Thiers (1988: 140, pl.1).

Specimens examined: Taveuni Island: S. \& T. Pócs 03299/BB, BC. 03280/BO, 03281/AC, 03288/ BO 03289/BM, 03290/E; Viti Levu Island: A.J. Fife 08037/B; Vanua Levu Island: M. von Konrat 0624-9 pp.

Cololejeunea falcata has been recorded from Madagascar, China (including Taiwan), Japan, Taiwan, Ogasawara-shoto (Bonin), Sri Lanka, Thailand, Vietnam, Cambodia, Peninsular Malaysia, Sumatra, Borneo, Philippines, Indonesia, New Guinea, Queensland, New Caledonia, and Mariana Is (cf. ELPT database).

13. Cololejeunea floccosa (Lehm. et Lindenb.) Schiffn., Conspectus Hepaticarum Archipelagi Indici: 243 (1898)

Basionym: Jungermannia floccosa Lehm. et Lindenb., Novarum et Minus Cognitarum Stirpium Pugillus 5: 26 (1833)

Type citation: Philippines, "Insula Luzon, ad portum Sorzogon, in foliis arborum. Sine nomine communicavit cl. Prof. Presl”.

Illustration: Benedix (1953: 134, pl. 4); Zhu \& So (2001: 343, fig. 128).

Specimens examined: Taveuni Island: S. \& T. Pócs 03289/EN.

Cololejeunea floccosa was reported by Piippo (1994) as a Paleotropical species.

14. Cololejeunea hirta Steph., Bulletin of Miscellaneous Information, Royal Gardens, Kew 1899: 125 (1899)

Type citation: Papua New Guinea. "Vanapa valley, 2000-4000 ft.”.

Illustration: Tixier (1985: 290, fig. 56)

Specimens examined: Taveuni Island: S. \& T. Pócs 03293/AF.

Cololejeunea hirta is a tropical Asian and South Pacific species, and has been reported from Vietnam, Peninsular Malaysia, Borneo, Indonesia (Java), New Guinea, Solomon Islands, and Tonga (Tixier 1985) as well as Hawaii (Mizutani 1961). However, Staples \& Imada (2007) treated Cololejeunea hirta as doubtful for Hawaii.

15. Cololejeunea huerlimannii Tixier, Nova Hedwigia 31: 773 (1979)

Type citation: New Caledonia, "Nouvelle Calédonie, Monts Koghis, S.E. du Mont Bouo, épiphylle sur feuilles de palmier, 830 m, 12.11.1950, Huerlimann 2093 (Holotype). Espèce dédiée au Dr. Huerlimann".

Illustration: Tixier (1979: 774, fig. 29); Tixier (1985: 247, fig. 30).

Specimens examined: Taveuni Island: S. \& T. Pócs 03279/BO.

Cololejeunea huerlimannii was previously known only from New Caledonia (Miller et al. 1983).

16. Cololejeunea inflectens (Mitt.) Benedix, Feddes Repertorium Specierum Novarum Regni Vegetabilis. Beiheft 134: 79 (1953)

Basionym: Lejeunea inflectens Mitt., Journal of the Proceedings of the Linnean Society 5: 117. (1861) 
Type citation: Sri Lanka, "In insula Ceylon, inter Macromitrias Horton Plains, Gardner" (Holotype: NY, isotype: FH).

Illustration: Benedix (1953: 134, pl. 30, as C. cilitilobula and C. peculiaris).

Specimens examined: Kadavu Island: S. \& T. Pócs 03304/AC, 03309/BE; Taveuni Island: S. \& T. Pócs 03282/BL, 03289/N, 03296/Z. Viti Levu Island: S. \& T. Pócs 03276/A T Pócs 08033/G, 08035/L, 08036/R.

Cololejeunea inflectens was reported by Piippo (1994) as a Paleotropical species , distributed from Madagascar and Seychelles to New Caledonia and Caroline Islands (Miller et al. 1983).

17. Cololejeunea kapingaensis H.A.Mill., The Bryologist 59: 170 (1956)

Type citation: Caroline Islands, "Werua islet, Kapingamarangi atoll, Caroline Islands, on bark of Pandanus 6 feet above soil level, mixed with other Hepaticae, W.A. Niering 38. July 2. 1954. Type specimen in the herbarium of the B. P. Bishop musuem, Honolulu; isotypes in the U. S. National Herbarium, Washington; Conservatoire Botanique, Genève; Dudley Herbarium, Stanford; and the herbarium of the author".

Illustration: Miller (1956: 172, fig. A-L).

Specimens examined: Viti Levu Island: S. \& T. Pócs 03253/W.

Cololejeunea kapingaensis is a Pacific species, including the Caroline and Marshall islands, and Australia (Pócs \& Streimann 2006).

18. Cololejeunea kulenensis Tixier, Bryophytorum Bibliotheca 27: 71 (1985)

Type citation: Sumatra, “Depok, leg. K.v. Goebel, n.2, Feb.1925”.

Illustration: Herzog (1932: 95, fig. 2a); Tixier (1985: 32, fig. 56).

Specimens examined: Kadavu Island: S. \& T. Pócs 03304/AB, 03308/H, 03309/BM. Taveuni Island: S. \& T. Pócs 03295/M. Viti Levu Island: $5.5 \mathrm{~km}$ NW of Suva city limits. Secondary forest over laterite. 19 Sept 1981 W.R. Buck 7192 (NY) Det B.M. Thiers.

Cololejeunea kulenensis has been reported for Cambodia, Peninsular Malaysia, and Sumatra (cf. ELPT database).

19. Cololejeunea lanciloba Steph., Hedwigia 34: 250 (1895)

Type citation: India, "Insulae Nicobaricae (Sulp. Kurz 3917)"

Illustration: Zhu \& So (2001: 278, fig. 106); Daniels, Kariyappa \& Daniel (2010: 292293, figs. 1-35).

Specimens examined: Kadavu Island: S. \& T. Pócs 03301/AE. Taveuni Island: S. \& T. Pócs 03295/M. Viti Levu Island: S. \& T. Pócs 03260/M.

Cololejeunea lanciloba is apparently a widespread paleotropical species (Piippo 1994).

20. Cololejeunea latilobula (Herzog) Tixier, Bryophytorum Bibliotheca 27: 156 (1985)

Basionym: Leptocolea latilobula Herzog in Handel-Mazzetti, Symbolae Sinicae 5: 54 (1930) 
Type citation: China [Sichuan], "Lebende Schoepfia jasminodora-Rinde in der wtp St. des mittelchin. Fl. bei Djindjischan nächst Loping, 1600 m. 12. VI. 1917 (10171)”

Illustration: Tixier (1985: 158 fig. 80); Asthana \& Srivastava (2003: 105, pl. 21).

Specimens examined: Kadavu Island: S. \& T. Pócs 03301/AE.

Cololejeunea latilobula was thought to be endemic to the Western Malesian region (Piippo 1994).

21. Cololejeunea longifolia (Mitt.) Benedix ex Mizut., Journal of the Hattori Botanical Laboratory 26: 184 (1963)

Basionym: Lejeunea longifolia Mitt., Journal of the Proceedings of the Linnean Society 5: 117 (1861)

Type citation: India. Sikkim Himalaya, 4-8000 ft., J.D. Hooker 1496 (Holotype: NY; isotype: BM, Zhu \& So 2001).

Illustration: Zhu \& So (2001: 309, fig. 117).

Specimens examined: Viti Levu Island: Naitasiri Prov.: Northern portion of Rairaimatuku Plateau, between Mt Tomanivi and Nasonggo, alt. 870-970 m. Aug-Sept 1947. A.C. Smith 5760 pp. (NY) Det. M. Fulford as Physocolea (Cololejeunea) leptolejeunoides Schiffn.

Cololejeunea longifolia was reported by Piippo (1994) as an Asian-Oceanian species.

22. Cololejeunea paucimarginata Tixier, Bryophytorum Bibliotheca 27: 100 (1985)

Type citation: Indonesia. “Java, Bogor, épiphylle sur Fougère, 250 m, 13. 4. 1969, Tixier 4338”. (Holotype, PC), 4339, 4440.

Illustration: Tixier (1985: 100, fig. 48).

Specimens examined: Taveuni Island: S. \& T. Pócs 03298/F.

Cololejeunea paucimarginata has previously been reported from Java (Tixier 1985). The taxon may be a form of C. raduliloba (R.-L. Zhu, pers. comm., in Söderström et al. 2010), but at least based on Fijian specimens, no transitional forms have been observed.

23. Cololejeunea pseudoserrata Tixier, Nova Hedwigia 31: 770 (1979)

Type citation: New Caledonia, "Nouvelle Calédonie, Rivière Bleue, 200 m. 24.7.1974. Schmid s.n. (Holotype)".

Illustration: Tixier (1979: 770: fig. 27); Tixier (1985: 283, fig. 52)

Specimens examined: Taveuni Island: S. \& T. Pócs 03279/CA, 03280/CM, 03285/BC, T. Pócs 08012/AD.

Cololejeunea pseudoserrata has previously been reported from New Caledonia (Miller et al. 1983).

24. Cololejeunea raduliloba Steph., Hedwigia 34: 251 (1895)

Type citation: Vietnam. Tonkin. Khang-Thuong (Ma Co), 9 Nov. 1885, Bon s.n. Holotype: G-1957 (Zhu \& So 2001).

Illustration: Tixier (1985: 105, fig. 51); Zhu (1995: 98); Isono (2009: 45-46, figs 3 \& 4). 
Specimens examined: Taveuni Island: S. \& T. Pócs 03285/B, 03293/AN. Viti Levu Island: S. \& T. Pócs 03267/A.

Cololejeunea raduliloba is a pantropical species with a distribution including, but not limited to the Seychelles, Madagascar, China, Korea, Japan, Vietnam, Borneo, Indonesia, Australia, New Caledonia, and Caroline Is (cf. ELPT database).

25. Cololejeunea schmidtii Steph., Botanisk Tidsskrift 24: 278 (1902)

Type citation: Thailand. Koh Chang, Klong Munsé \& Nipple, 2000 ft., Schmidt. Holotype: G-14217 (Zhu \& So 2001).

Illustration: Benedix (1953: 134, pl. 16); Zhu \& So (2001: 334, fig. 125).

Specimens examined: Taveuni Island: S. \& T. Pócs 03279/AW, 03280/CD, 03288/CV, 03289/CW. Viti Levu Island: S. \& T. Pócs 032069/K, 03273/CH, 03274/BB.

Cololejeunea schmidtii is a Southeast Asiatic species distributed from Sri Lanka to Japan and Papua New Guinea (Pócs \& Streimann 2006).

26. Cololejeunea sintenisii (Steph.) Pócs, in Dauphin et al., Cryptogamie, Bryologie 29: 235.2008

Basionym: Aphanolejeunea sintenisii Steph., Species Hepaticarum 5: 861 (1916)

Type citation: Puerto Rico, "Sintenis No. 136".

Illustration: Schuster (1980: 1304, fig. 771, as Aphanolejeunea ephemeroides; Allorge \& Jovet-Ast (1950: 21, figs 1 \& 2, as Aphanolejeunea teotonii).

Specimens examined: Viti Levu Island: T. Pócs 08036/H.

Cololejeunea sintenisii is a pantropical species (Pócs \& Bernecker 2009).

27. Cololejeunea societatis Tixier, Bauhinia 8: 230 (1987)

Type citation: Tahiti, “T 1182 a (Holotypus PC, isotypus Hb. Hürlimann Basel) epiphyll an Strauch nahe der Wasserfassung ob der landwirtschftlichen Versuchsstation auf der Halbinsel Taiarapu. 13. II. 1952, leg H[ans] H[ürlimann]”.

Illustration: Hürlimann (1987: 231, fig. 4).

Specimens examined: Taveuni Island: S. \& T. Pócs 03289/BJ, BO.

Cololejeunea societatis has previously only been reported for Society Is (cf. ELPT database).

28. Cololejeunea stylosa (Steph.) Steph. ex Mizut., Journal of the Hattori Botanical Laboratory 28: 109 (1965)

Basionym: Lejeunea stylosa Steph., Hedwigia 27: 289 (1888)

Type citation: Philippines, "Insula Luzon, leg. Micholitz, 1886".

Illustration: Zhu \& So (2001: 271, fig. 104).

Specimens examined: Viti Levu Island: S. \& T. Pócs 03252/BD, 03276/H.

Cololejeunea stylosa has been recorded from China, Japan, Nansei-Shoto (Ryu-Kyu Is), Taiwan, Ogasawara-shoto (Bonin), Kazan-retto (Volcano), Thailand, Laos, Vietnam, 
Cambodia, Peninsular Malaysia, Philippines, Queensland, and Mariana Is (cf. ELPT database).

29. Cololejeunea tridentata Tixier, Bryophytorum Bibliotheca 27: 83 (1985)

Type citation: Malaysia, “Johore, Mont Ophir, épiphylle dans les brousses au bord du lac de retenue, 300 m, 20. 4. 1972, Tixier 6255 (Holotype, PC)”.

Illustration: Tixier (1985: 83, fig. 39).

Specimens examined: Taveuni Island: S. \& T. Pócs 03288/CX.

Cololejeunea tridentata was previously known from Borneo and Malaysia (Eggers et al. 1998).

30. Cololejeunea wightii Steph., Hedwigia 34: 253 (1895)

Type citation: [Malaya] Pulo Penang, s. d., Wallich s. n., ex hb Schiffner in hb G. Holotype: G-22054 (Zhu \& So 1999b).

Illustration: Miller (1968:254, fig. 37); Tixier (1979: 759, fig. 20 as Cololejeunea cuspidata Steph.); Tixier (1985: 249, fig. 31 as C. wightii; 1985: 250, fig. 32 as C.cuspidata); Thiers (1988: 136, pl. 9), Zhu \& So (1999: 169, fig. 3); Miller et al. (1963a: pl. 21).

Specimens examined: Kadavu Island: S. \& T. Pócs 03300/J, 03306/C. Taveuni Island: S. \& T. Pócs 0303291/B, 03292/G, 03293/D. Viti Levu Island: S. \& T. Pócs 03252/W.

Cololejeunea wightii has a Pantropical distribution (Zhu \& So 1999), with reports from Seychelles, China, ?India, Thailand, Cambodia, Peninsular Malaysia, New Guinea, Queensland, Norfolk I, New Caledonia, Tonga, Samoa, and Caroline Is (cf. ELPT database).

31. Colura brevistyla Herzog, Beihefte zum Botanischen Centralblatt 38: 331 (1921)

Fig. 1c \& 2a,b

Type citation: Sri Lanka, "Ceylon: ohne näheren Fundortsvermerk, leg. Herzog II. 1906".

Illustration: Jovet-Ast (1953: 277, fig. 47).

Specimens examined: Viti Levu Island: T Pócs 08032/C, 08034/N, 08036/G, V. Taveuni Island: S. \& T. Pócs 03288/BE.

Colura brevistyla has been reported from Sri Lanka, Vietnam Philippines, and the Mariana Islands (cf. ELPT database). The specimens from Fiji have stronger lobe dentition than the type.

32. Colura tenuicornis (A.Evans) Steph., Species Hepaticarum 5: 942 (1916) Fig. 2c Basionym: Colurolejeunea tenuicornis A.Evans, Transactions of the Connecticut Academy of Arts and Sciences 10: 455 (1900)

Type citation: Hawaii. Oahu. Nuuanu (Cooke), on leaves of a fern, A. W. Evans s.n. \& s.d. Holotype: YU. (Zhu \& So 2001).

Illustration: Jovet-Ast (1953: 249, fig. 30); Zhu \& So (2001: 234, fig. 89).

Specimens examined: Viti Levu Island: T. Pócs 08023/AA. 


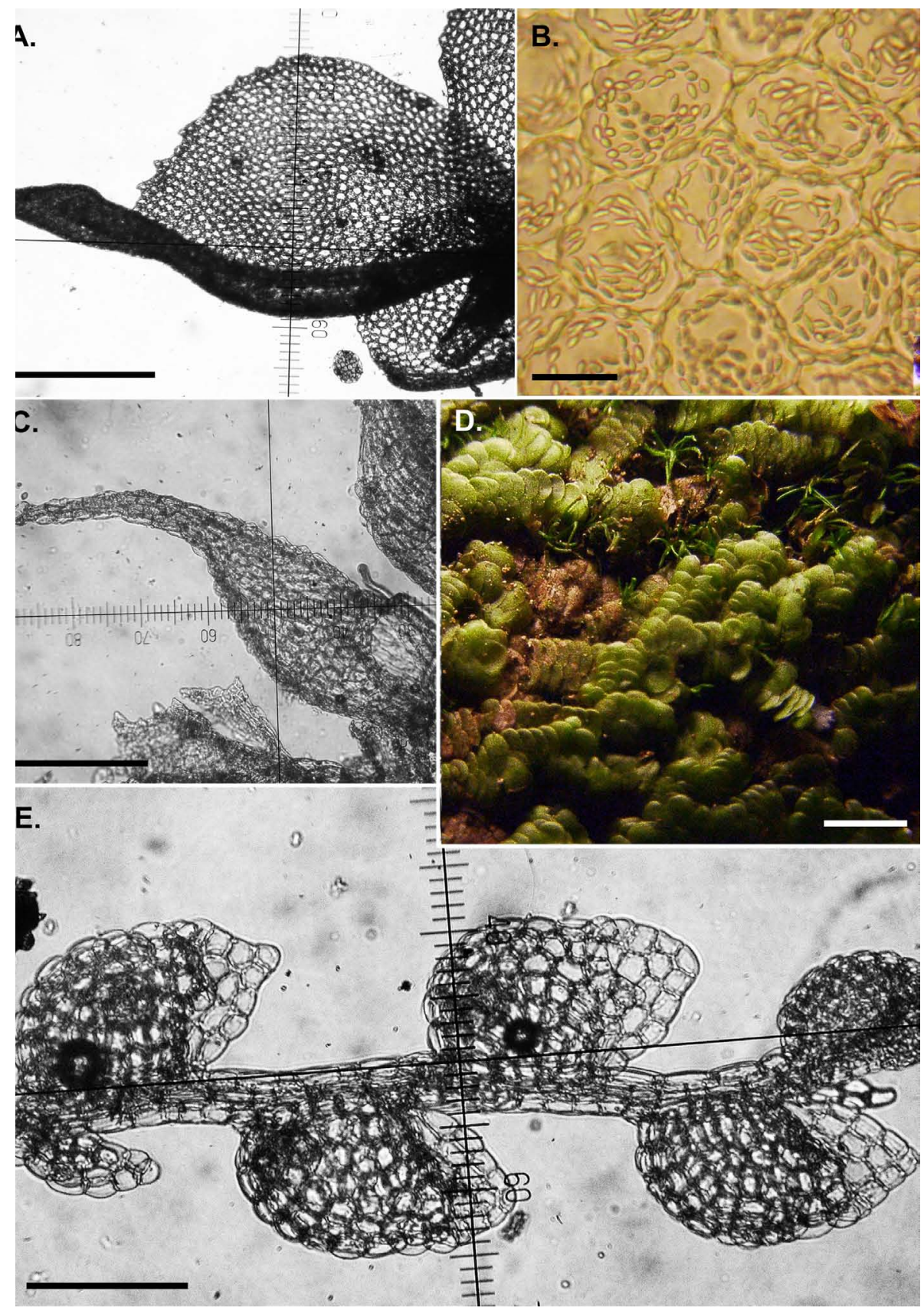

Fig. 2. A, B, Colura brevistyla (T. Pócs $08036 / V$ ). A, ventral lobe, scale bar $500 \mu \mathrm{m}$; B, oil bodies and cell anatomy, scale bar $20 \mu \mathrm{m}$; C, Colura tenuicornis, lobe and lobule (S. \& T. Pócs 03274/ $A O$ ), scale bar $500 \mu \mathrm{m}$; D, Phaeolejeunea inermis, habit on decaying wood (S. \& T. Pócs 03300/ $A G$ ), scale bar $2 \mathrm{~mm}$; E, Lejeunea exilis, ventral view (S. \& T. Pócs 03289/BY), scale bar $100 \mu \mathrm{m}$. 


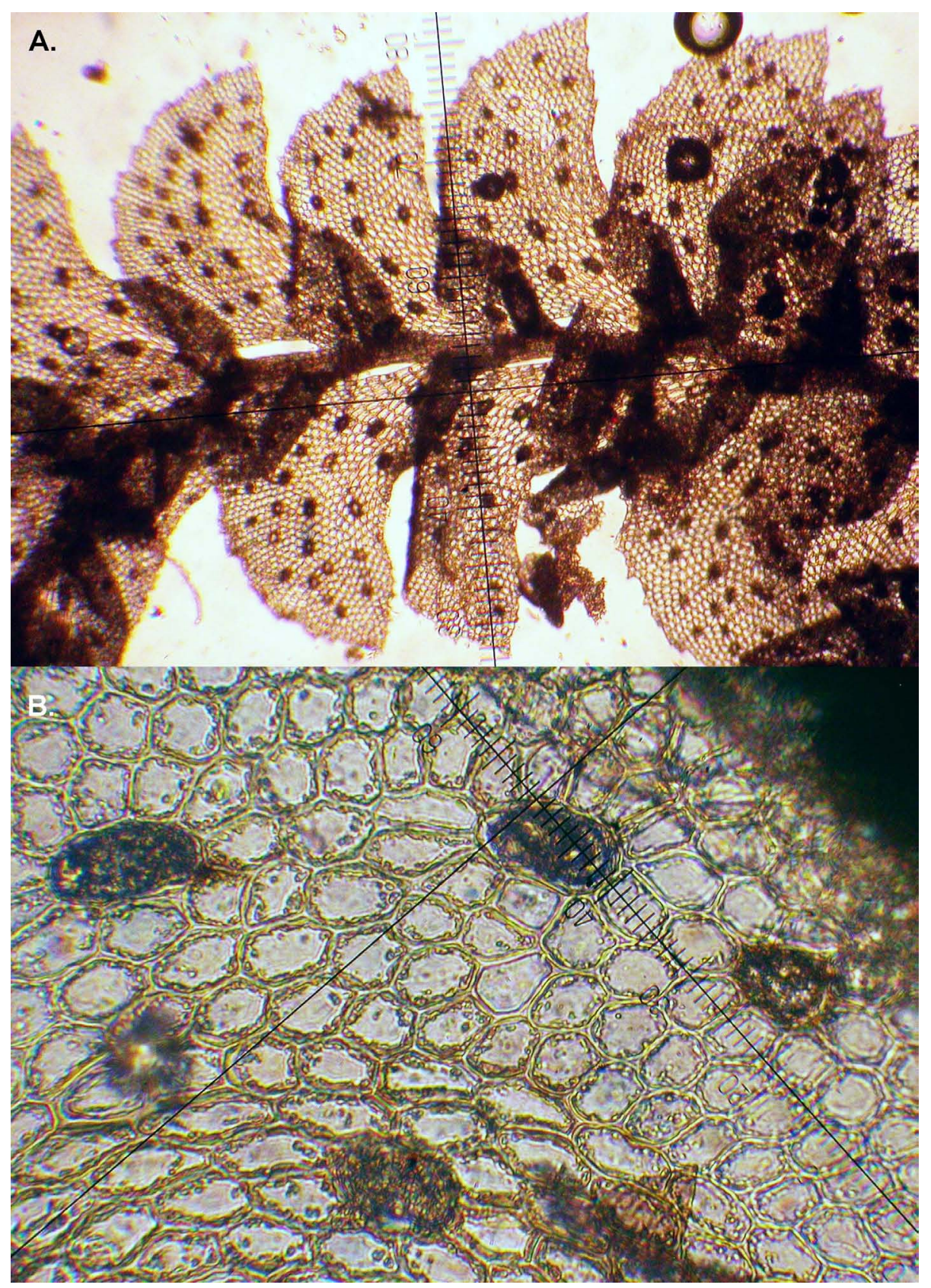

Fig. 3. A, B, Drepanolejeunea tricornua, (S. \& T. Pócs 03280/BT). A, ventral view; B, median cells of leaf lobe. 
Colura tenuicornis was reported by Piippo (1994) to be a Paleotropical species, excluding Australia.

33. Drepanolejeunea tricornua Herzog, Annales Bryologici 9: 124 (1936) Fig. 3a,b

Type citation: Indonesia. Seram, "S.O. Ceram. auf Oldenhamia auriculata, bei Wai Tok. 0-100 m, Kornassie no. 1055/a, 27.II.18 (Herb., H. B. Bog. no 4323)”.

Illustration: Herzog (1936: 124, fig. 6); Mizutani (1990: 371, fig.2).

Specimen examined: Taveuni Island: S. \& T. Pócs 03280/BS \& BT.

Drepanolejeunea tricornua has been recorded from Borneo, Java, Seram and New Guinea (Mizutani 1990).

34. Kurzia gonyotricha (Sande Lac.) Grolle, Revue Bryologique et Lichénologique 32: 167 (1964)

Basionym: Lepidozia gonyotricha Sande Lac. Nederlandsch Kruidkundig Archief. Verslangen en Mededelingen der Nederlandsche Botanische Vereeniging 3: 521 (1851)

Type citation: Indonesia. Java, leg. Junghuhn. Source: IH online.

Illustration: Piippo (1985 177, fig 5f).

Specimen examined: Vanua Levu Island: Waisali Dakua National Trust Forest Reserve. On dead rotting log and humus at track margin, with Telaranea and Bazzania. M. von Konrat, J. E. Braggins, \& A. Naikatini 06/8-8(1a). July 2006. Det. E. Brown.

Kurzia gonyotricha has been reported from Japan, Ryukyu, Taiwan, China (8 provinces), Thailand, Peninsular Malaysia, Sumatra, Java, Borneo, Sulawesi, Moluccas, Philippines, New Guinea, and New Caledonia (cf. ELPT database).

35. Lejeunea alata Gottsche, Synopsis Hepaticarum 3: 406 (1845)

Type citation: Lectotype ("Grolle 1977; der Holotypus ist in B verbrannt"): Maskarenen, ,in Memecylo cordato; Sieber, Fl. Mixt. [Exs.] no. 170, a cl. Sonder illatum', STR. Isolectotype Grolle 1977): G-17836, S, W (Lindenb. Hep. 6876).

Illustration: Zhu \& So (2001: 138, fig. 53).

Specimens examined: Taveuni Island: S. \& T. Pócs 03280/CS. Viti Levu Island: S. \& T. Pócs 03273/R.

Lejeunea alata has been recorded from Africa: Tanzania and Indian Ocean Islands; S-China, Vietnam, Malaysia, Java, Sumatra, New Guinea, Samoa, and Polynesia (mostly after Zhu \& So 2001).

36. Lejeunea anisophylla Mont., Annales des Sciences Naturelles; Botanique, sér. 2 19: $263(1843)$

Type citation: Hawaii. "ad cortices inter uscos in insulis Sandwich". Gaudichaud s.n. \& s.d. Holotype: PC, Isotypes: BM, G (Zhu \& So 2001).

Illustration: Jones (1972: 38-39 as L. caespitosa); Zhu \& So (2001: 157 fig. 62).

Selected specimens examined: Kadavu Island: S. \& T. Pócs 03301/AL. Taveuni Island: T Pócs 08021/H. Viti Levu Island: S. \& T. Pócs 03252/Y 
In Fiji, the species is very widespread, with more than 60 specimens collected on Kadavu, Taveuni, and Viti Levu on very different substrates. Lejeunea anisophylla is a Palaeotropic species common in Africa (Pócs 2010), tropical and subtropical Asia, Australia and Oceania eastwards to Tahiti and Hawaii (Zhu \& So 2001).

37. Lejeunea apiculata Sande Lac., in Dozy (ed.), Nederlandsch Kruidkundig Archief. Verslagen en Mededelingen der Nederlandsche Botanische Vereeniging 3: 421 (1854) [1855]

Synonym: Stenolejeunea apiculata (Sande Lac) R.M.Schust. Beihefte zur Nova Hedwigia 9: 144.1963

Type citation: Indonesia. Java, "Javam. TEYSM. in Herb. Dz. et Mb. In monte Salak. ZOLLINGER coll. sub n. 3560. Herb. JUNGH”.

Illustration: Eifrig (1937: 100, fig. XII/2 as Taxilejeunea apiculata).

Specimens examined: Viti Levu Island: S. \& T. Pócs 03274/BQ, BV.

Lejeunea apiculata has been recorded from Shaanxi, Shanxi, Guangdong, Hong Kong, Hainan, Japan, Nansei-Shoto (Ryu-Kyu Is), Taiwan, Sri Lanka, Vietnam, Cambodia, Nicobar Is, Sumatra, Borneo, Philippines, Sulawesi, Java, Australia (Queensland), and New Caledonia (cf. ELPT database).

38. Lejeunea cocoes Mitt., Journal of the Proceedings of the Linnean Society. Botany 5: 114. (1860) [1861]

Type citation: Sri Lanka, "Ad truncos Cocoes nuciferae, Balagom, Ceylon, Gardner (No. 1499)".

Illustration: Mizutani 1963: 177, fig s 10-18.

Specimens examined: Viti Levu Island: S. \& T. Pócs 03255/A, 03259/Z. Taveuni Island: S. \& T. Pócs 03283/T, 03285/BK, 03287/B, 03293/U,03294/C. Kadavu Island: S. \& T. Pócs 03300/G, 03308/D.

Lejeunea cocoes is known from Diego Garcia, Chagos, Sri Lanka, S-India, S-China, Java, and Sarawak. The species is a new record for the Pacific.

39. Lejeunea exilis (Reinw., Blume et Nees) Grolle, Journal of the Hattori Botanical Laboratory 46: 353 (1979)

Fig. 2e

Basionym: Jungermannia exilis Reinw., Blume et Nees, Nova Acta Physico-Medica Academiae Caesareae Leopoldino-Carolinae Naturae Curiosorum Exhibentia Ephemerides sive Observationes Historias et Experimenta 12: 227 (1824) [1825]

Type citation: Indonesia. Java (Neotype designated by Bischler et al. 1962: STR sub. Microlejeunea erectifolia, isoneotype: W Lindenb. Hep. 6724 p.p. sub. Microlejeunea cucullata, PC-Mont., S) (Grolle 1979).

Synonym: Lejeunea microstipula Steph. Hedwigia 29: 89, 139, XVI (1890) "MicroLejeunea" syn. nov.

Type citation: Indonesia. Java, hb. Lindenberg n. 6721.

Grolle (1980) in his account on the liverworts of Samoa reported Lejeunea microstipula Steph. from Upolu Island, based on a specimen collected by Schultze-Motel 3640 and 
3760/b and stated that L. microstipula belonged to subgen. Byssolejeunea, along with its closest relative, Lejeunea exilis, of which differs only by its acute leaf lobe. Zhu \& Grolle (2003) in their detailed study on Lejeunea exilis described the species having very variable leaf lobes, i.e. "leaf lobes ovate, ovate-lanceolate or irregularly triangularovate" with "apex acute, short acuminate, rounded, obtuse, rounded-obtuse, or obtuseacute, flat, rarely incurved". This variability was also illustrated in their figures, where the lobe apex varied from acute through triangular-obtuse to rounded, even within the same plant. The same variability was illustrated by Stepani in his Icones (Stephani 1985: 007759, 007760), where the type of Lejeunea microstipula has an acute lobe while another specimen collected by Paterson has ovate lobes with a rounded apex. This variability can be observed with Fijian specimens, therefore we believe the two taxa cannot be treated as separate species.

Illustration: Zhu \& Grolle (2003: 103,104, figs. 1, 2).

Specimens examined: Taveuni Island: S. \& T. Pócs 03269/P, 03273/CR, 03278/M, 03280/CO, 03286/AT, 03288/AD, 03289/BY, 03290/BA, T. Pócs 08013/AH. Viti Levu Island: S. \& T. Pócs 03271/AF, 03272/B, 03273/AG, 03274/AB, AN, 08029/C pp, 08029/O.

Lejeunea exilis was reported by Piippo (1994) as an Asian-Oceanian species; with records from Réunion, Shaanxi, Anhui, Taiwan, Ogasawara-shoto (Bonin), Kazanretto (Volcano), India, Vietnam, Peninsular Malaysia, Sumatra, Borneo, Philippines, Moluccas, Java, New Guinea, Australia, Society Is, Caroline Is, and Mariana Is. (cf. ELPT database). Grolle (1980) recorded Lejeunea exilis for Samoa as Lejeunea microstipula Steph.

40. Lepidolejeunea borneensis (Steph.) R.M.Schust., Phytologia 45: 425 (1980)

Basionym: Hygrolejeunea borneensis Steph., Species Hepaticarum 5: 557 (1914)

Type citation: Indonesia, "Borneo occidentalis".

Illustration: Piippo (1986: 37, fig. 10).

Specimens examined: Viti Levu Island: T Pócs 08036/JB, AA. Taveuni Island: S. \& T. Pócs 08036/ $J B, A A$.

Lepidolejeunea borneensis has previously been reported for Malay Peninsula, Borneo, and Caroline Is (cf. ELPT database).

41. Lopholejeunea hispidissima Steph., Species Hepaticarum 5: 80 (1912)

Type citation: New Caledonia. Franc s.n. Holotype: G, Isotype: FH (Thiers \& Gradstein 1989).

Illustration: Thiers \& Gradstein (1989: 29, fig. 9).

Specimens examined: Taveuni Island: S. \& T. Pócs 03292/K. Viti Levu Island: S. \& T. Pócs 03263/G. Det. A. Sass-Gyarmati.

Lopholejeunea hispidissima has been reported for Australia (Queensland), New Caledonia, and Vanuatu (Sass-Gyarmati 2002). 
42. Microlejeunea lunulatiloba Horik., Journal of Science of Hiroshima University, Series B, Division 2 (Botany) 1: 27 (1931)

Type citation: Bonin Islands [Ogasawara Gunto] "Bonin: Insl. Chichijima (Y. Horikawa, no. 1962b-type,... July 1930)”. Source: IH online.

Illustration: Miller et al. (1963b, pl. $131 \mathrm{f}-1$ ).

Specimens examined: Viti Levu Island: S. \& T. Pócs 03259/AA.

Microlejeunea lunulatiloba is a Pacific species known from the Bonin, Caroline, Marshall, Mariana and Hawaiian islands (Miller et al. 1963a, Miller et al. 1983).

43. Mnioloma novaezelandiae J.J.Engel, Cryptogamie, Bryologie 27: 111 (2006)

Type citation: New Zealand, South Island, Southland Land District, Fiordland Natl. Park, Charles Sound, south side of Gold Arm, 340 m, 8 April 2002, Renner CMS J156 T/10 (Holotype, F; Isotypes, AK, CHR) (Engel 2006).

Illustration: Engel \& Glenny (2008: 540, fig. 123).

Specimens examined: Viti Levu Island: T. Pócs 08028/F.

Mnioloma novaezelandiae has previously only been recorded from New Zealand. A report for Queensland by Renner \& Braggins (2006) was based on an erroneous identification (Renner \& Brown 2008), referring it to Mnioloma cf. fuscum. It is apparent that the complex needs revision in Australasia and Oceania.

44. Phaeolejeunea inermis (Steph.) Mizut., Journal of the Hattori Botanical Laboratory 31: 134 (1968)

Fig. 2d

Basionym: Lopholejeunea inermis Steph., Species Hepaticarum 5: 92 (1912)

Type citation: Papua New Guinea. Milne Bay Prov.: Samarai, Fitzgerald s.n. Holotype: G-12642 (Gradstein et al. 2002).

Illustration: Mizutani (1968: XX, Fig II); Mizutani (1968: 133, fig.II/9-15).

Specimens examined: Kadavu Island: S. \& T. Pócs 03300/AG.

Phaeolejeunea inermis was previously known only from the type collected in Papua New Guinea (Mizutani 1968, Gradstein et al. 2002). Mizutani suggested, based on the scanty type specimen, that it is a poorly developed form of P. latistipula (Schiffn.) Mizut. However, the abundant material from Fiji, with seemingly stable characters, supports the independent identity of $P$. inermis.

45. Plagiochila bicornuta Steph., Botanische Jahrbücher für Systematik, Pflanzengeschichte und Pflanzengeographie 23: 305 (1897)

Type citation: Samoa. R[einecke] 55. Holotype: G (So 2000).

Illustration: So (2000: 227, fig. 2).

Specimens examined: Taveuni Island: T. Pócs 08013/AE. 

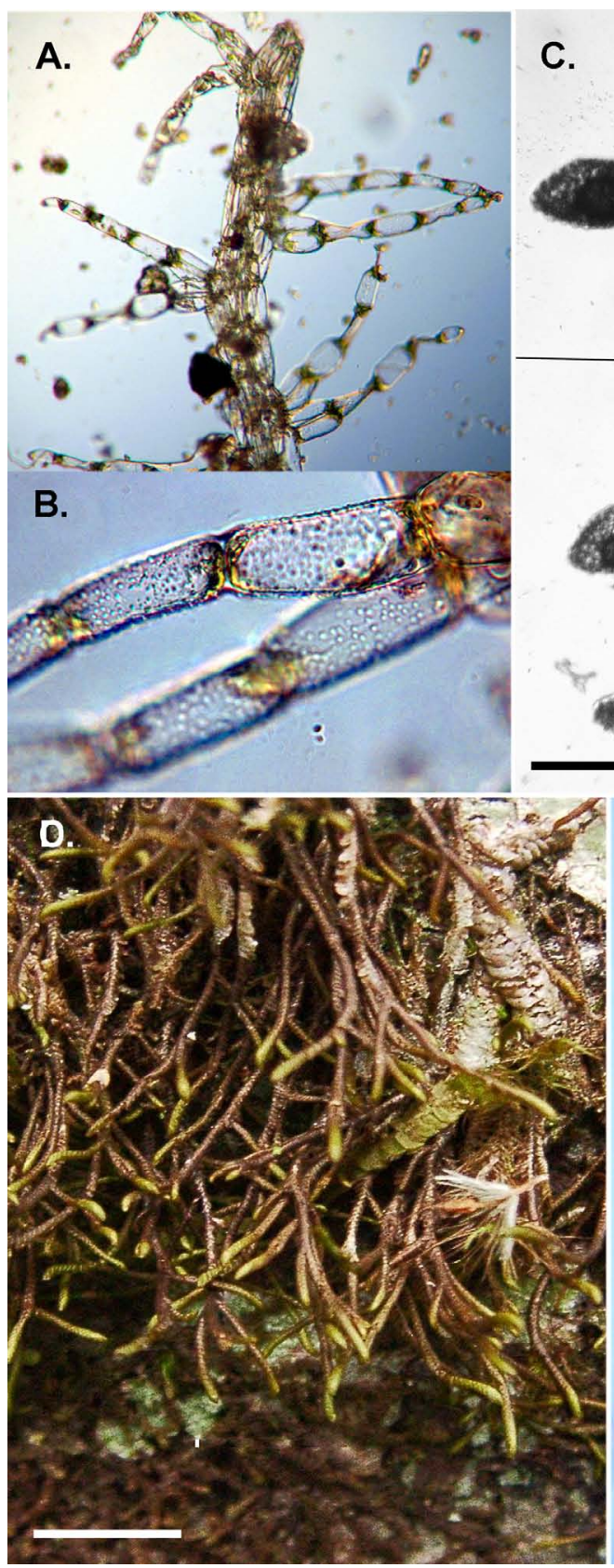

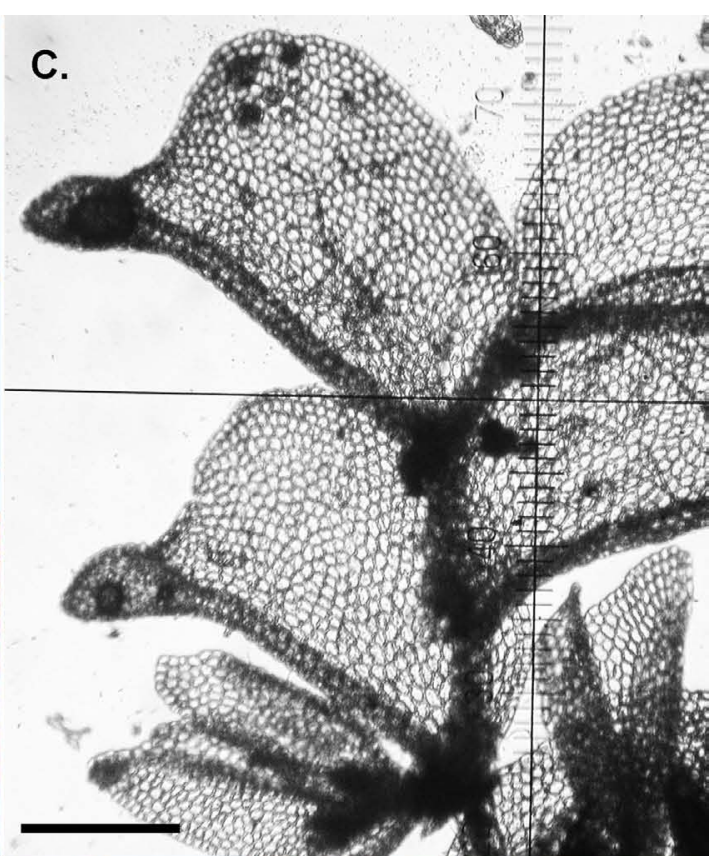

E.

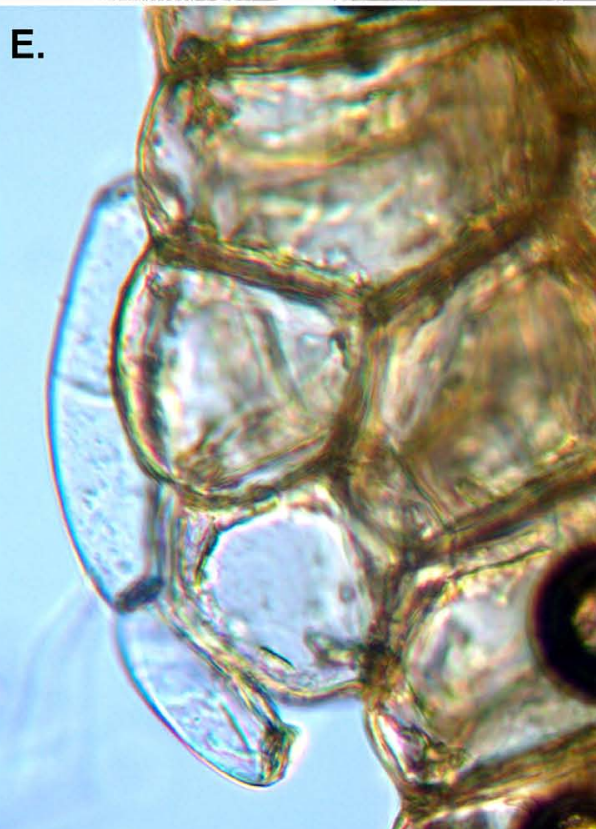

Fig. 4. A, B, Telaranea rosarioana (M. von Konrat, J. E. Braggins \& A. Naikatini 06/7-13). A, habit with deeply bilobed leaves, B, cuticle conspicuously papillose; C, Colura acroloba, (S. \& T. Pócs

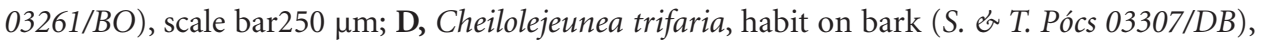
scale bar $1 \mathrm{~cm}$. E, Zoopsis liukiuensis (M. von Konrat, J. E. Braggins, \& A. Naikatini 06/8-8(1a). 
Plagiochila bicornuta has been recorded from Borneo, Philippines, Moluccas, Java, New Guinea, New Caledonia, Vanuatu, Samoa, and Caroline Is (cf. ELPT database).

46. Pleurozia conchifolia (Hook. et Arn.) Austin, Bulletin of the Torrey Botanical Club 5: 16 (1874)

Basionym: Jungermannia conchifolia Hook. et Arn., The Botany of Captain Beechey's Voyage: 110 (1832)

Type citation: Sandwich Is. 1826, Beechey (Holotype: BM, Isotypes: BM, FH, H, NY, PC) (Thiers 1993).

\section{P. conchifolia var. conchifolia}

Fig. 1c

Illustration: Thiers (1993: 527, figs. 14-18).

Specimens examined: Taveuni Island: S. \& T. Pócs 03279/N.

Pleurozia conchifolia var. conchifolia has been recorded from Burma (Myanmar), Malay Peninsula, Sumatra, Borneo, Sulawesi, Moluccas, New Guinea, Solomon Islands, and Hawaii (cf. ELPT database).

47. Telaranea rosarioana H.A.Mill., Journal of Bryology 14: 240 (1986) Fig. 4a,b

Type citation: Vanuatu. "Espiritu Santo Island, summit of Mt Patliu above Narango, 25 July 1984. H. A. Miller 15993 in admixture with Symphyogyna and Leucophanes (paratype)".

Illustration: Miller (1986: 241, fig.4).

Specimens examined: Viti Levu: Rairaimatuku Plateau, on a summit with disturbed stunted forest with a telecommunication tower, ca. $15 \mathrm{~km} \mathrm{~S}$ of Monasavu Dam, at $1260 \mathrm{~m}, \mathrm{~S} 17^{\circ} 47^{\prime} 31^{\prime \prime}, \mathrm{E}$ $178^{\circ} 01^{\prime} 13.9^{\prime \prime}$, on very old, rotted tree fern trunk, M. von Konrat, J. Braggins \& A. Naikatini 06/713, July 2006. Det. M. von Konrat \& T. Pócs.

Telaranea rosarioana was previously known only from the type locality in Vanuatu (Engel \& Smith Merrill 2004). Engel \& Smith Merrill (2004) mistakenly stated that this taxon was endemic to Fiji.

48. Zoopsis liukiuensis Horik., Journal of Science of Hiroshima University, Series B, Division 2 (Botany) 1: 65 (1931)

Fig. 4e

Type citation: Japan. Liukiu, Okinawa, Horikawa 2620. Source: IH online.

Illustration: Schuster (2000: 406-407, figs. 168-169).

Specimens examined: Vanua Levu Island: Waisali Dakua National Trust Forest Reserve. On dead rotting log and humus at track margin, with Telaranea and Bazzania. M. von Konrat, J. E. Braggins \& A. Naikatini 06/8-8(1a). July 2006. Det. M. von Konrat \& J. Braggins. Viti Levu Island: on the ridge of Rairaimatuku Plateau, ca. $2 \mathrm{~km}$ E of Monasavu Dam, on the SW side of a summit at 1050-1070 m alt. S $17^{\circ} 45^{\prime} 21^{\prime \prime}$, E $178^{\circ} 04^{\prime} 09^{\prime \prime}$. Shady, very wet stunted forest dominated by Dicksonia brackenridgei. On dead decaying matter at base of tree. M. von Konrat with T. Pócs, J. Braggins, E. Brown, M. Renner \& A. Naikatini 08/2-18, 16 Apr. 2008, Det. M. von Konrat \& J. Braggins. 
Zoopsis liukiuensis is known from S Japan, S China, Philippines, Malay Peninsula, Sumatra, Java, Borneo, New Guinea, Queensland, Vanuatu, and Samoa (Piippo 1984).

\section{Extended distribution records}

Distribution is extended by reporting new islands within Fiji for the following 39 taxa.

49. Acrolejeunea pycnoclada (Taylor) Schiffn., in Engler \& Prantl, Die natürlichen Pflanzenfamilien 1(3): 128 (1895)

Basionym: Ptychanthus pycnocladus Taylor, London Journal of Botany 5: 385 (1846)

Type citation: Malaysia, “On bark. Malacca. Hook. Herb.”.

Acrolejeunea pycnoclada subsp. pycnoclada

Illustration: Gradstein et al. (2002: 8, fig. 3).

Specimens examined: Kadavu Island: S. \& T. Pócs 03300/U, TA.

Acrolejeunea pycnoclada subsp. pycnoclada is a new report for the island of Kadavu. This species has a paleotropical distribution (Piippo 1994). Det. A. Sass-Gyarmati.

50. Archilejeunea planiuscula (Mitt.) Steph., Species Hepaticarum 4: 731 (1911)

Basionym: Lejeunea planiuscula Mitt., Journal of the Proceedings of the Linnean Society. Botany 5: 111 (1860) [1861]

Type citation: Burma, "Pegu ad Rangoon, inter L. ungulatae et Meteorii squarrosi caespites, M'Clelland'.

Illustration: Thiers \& Gradstein (1989: 9, fig. 1; Gradstein et al. (2002: 12, fig. 5).

Specimens examined: Kadavu Island: S. \& T. Pócs 03310/D. Taveuni Island: S. \& T. Pócs 03282/P. Det. A. Sass-Gyarmati.

Archilejeunea planiuscula is a new report for the islands of Kadavu and Taveuni. This species is known from many localities in the Indo-Pacific region (Sass-Gyarmati 2003).

51. Caudalejeunea reniloba (Gottsche) Steph., Species Hepaticarum 5: 16 (1912)

Basionym: Phragmicoma reniloba Gottsche, Synopsis Hepaticarum 3: 301 (1845).

Type citation: Indonesia, Java, Banten, Blume s.n. (G, lectotype designated by Thiers \& Gradstein 1989).

Illustration: Gradstein et al. (2002: 19, fig. 9).

Specimens examined: Taveuni Island: S. \& T. Pócs 03288/F, BP. Det. A. Sass-Gyarmati.

Caudalejeunea reniloba is a new report for the island of Taveuni. It is widespread in tropical regions, including Oceania and Asia (Zhu \& So 2001) Philippines, New Guinea, Indonesia, Oceania, Mascarenes, and Australia (northern Queensland) Sass-Gyarmati (2002). 
52. Ceratolejeunea belangeriana (Gottsche) Steph., Species Hepaticarum 5: 396 (1913)

Basionym: Lejeunea belangeriana Gottsche, Synopsis Hepaticarum 3: 398 (1845)

Type citation: Mauritius, "Insula Franciae (Belanger in Hb L.)”.

Illustration: Bonner (1953: 169, fig. 8).

Specimens examined: Kadavu Island: S. \& T. Pócs 03300/AP.

Ceratolejeunea belangeriana is a new report for the island of Kadavu. This species has a paleotropical distribution (Piippo 1994).

53. Cheilolejeunea ceylanica (Gottsche) R.M.Schust. et Kachroo in Kachroo \& Schuster, Journal of the Linnean Society. Botany 56: 509 (1961)

Basionym: Lejeunea ceylanica Gottsche, Synopsis Hepaticarum 3: 359 (1845)

Type citation: Sri Lanka, "Insula Ceylon. Inter Radulam javanicam cum Lej. Lindenbergii (ex Hb. Hk.) inveni”.

Illustration: Zhu \& So (2001: 118, fig. 47).

Specimens examined: Taveuni Island: S. \& T. Pócs 03288/DQ. Kadavu Island: S. \& T. 03304/K.

Cheilolejeunea ceylanica is a new report for the islands of Taveuni and Kadavu. This species has a paleotropical distribution (Piippo 1994).

54. Cheilolejeunea intertexta (Lindenb.) Steph., Bulletin de l'Herbier Boissier, sér. 1 5: 79 (1897)

Basionym: Lejeunea intertexta Lindenb., Synopsis Hepaticarum 3: 379 (1845)

Type citation: Karolinen "in Ualan, Ulleay et Lugnor Insul. Carol." leg. Mertens, Lindenb. hep. 6653/a, c. per + $\widehat{o}$ (Lectotype: W, designated by Grolle 1979).

Illustration: Mizutani (1982: 171, fig. 9).

Specimens examined: Kadavu Island: S. \& T. Pócs 03303/A. Taveuni Island: S. \& T. Pócs 03276/C, 03283/L, 03285/E, 03287/A, C, E, 03294/E.

Cheilolejeunea intertexta is a new report for the island of Kadavu. This species has a paleotropical distribution (Piippo 1994).

55. Cheilolejeunea trapezia (Nees) R.M.Schust. et Kachroo, Journal of the Hattori Botanical Laboratory 24: 282 (1961)

Basionym: Jungermannia trapezia Nees, Enumeratio Plantarum Cryptogamicarum Javae: 41 (1830)

Type citation: Indonesia. Java, "in Collemate bullato semcl inventa, absque fructificatione".

Illustration: Mizutani (1980: 328, fig. 5 as C. longiloba); Zhu \& So (2001: 120, fig. 48, as C. imbricata); Stephani (1985: 001792) (as Cheilolejeunea inaequitexta).

Specimens examined: Taveuni Island: S. \& T. Pócs 03293/V. Kadavu Island: S. \& T. Pócs 03293/V. 
Zhu \& Grolle (2004) studied Cheilolejeunea trapezia in detail, noting that it was the correct name for a species commonly referred to as C. imbricata (Nees) S. Hatt. Zhu et al. (2002) stated that C. longiloba Steph. ex G. Hoffm. is also synonym of C. trapezia. Zhu \& Grolle (2004) stated C. trapezia was a highly variable species and noted its main characters. It is a widely distributed in tropical and subtropical regions in Asia and Oceania. Cheilolejeunea trapezia is a new report for the islands of Taveuni and Kadavu.

56. Cheilolejeunea trifaria (Reinw., Blume et Nees) Mizut., Journal of the Hattori Botanical Laboratory 27: 132 (1964)

Fig. 4d

Basionym: Jungermannia trifaria Reinw., Blume et Nees, Nova Acta Physico-Medica Academiae Caesareae Leopoldino-Carolinae Naturae Curiosorum Exhibentia Ephemerides sive Observationes Historias et Experimenta 12: 226 (1825)

Type citation: Indonesia. Java, “ad terrram. caespitosa. Blume. V. c. cal.”

Illustration: Mizutani (1982: 169, fig. 8).

Specimen examined: Kadavu Island: 03305/C, 03300/AU, 03307/K, DB.

Cheilolejeunea trifaria is a new report for the island of Kadavu. This species is widely distributed from tropical Africa to the Indomalesian and Australasian regions. Piippo (1994) noted the taxon as a transpacific species.

57. Cololejeunea ceatocarpa (Ångstr.) Steph., Bulletin de l'Herbier Boissier, sér. 1 5: 842 (1897)

Basionym: Lejeunea ceatocarpa Ångstr., Öfversigt af Förhandlingar: Kongl. Svenska Vetenskaps-Akademien 29(4): 27 (1872) [1873]

Type citation: Hawaii. Honolulu, "in caulibus foliisque Cryptopodii bartramioides".

Illustration: Tixier (1985: 269, fig. 44).

Specimens examined: Taveuni Island: S. \& T. Pócs 03284/L.

Cololejeunea ceatocarpa is a new report for the island of Taveuni. Recorded elsewhere from Réunion, Bangladesh, Vietnam, New Caledonia, Fiji, Tonga, and Hawaii (cf. ELPT database).

58. Cololejeunea cordiflora Steph., Hedwigia 34: 246 (1895)

Type citation: New Caledonia, “(Balansa 3694) Herb. Bescherelle”.

Illustration: Zhu \& So (2001: 242, fig. 93).

Specimens examined: Taveuni Island: S. \& T. Pócs 03279/AZ, 03281/AB, 03282/BB, 03285/BA, 03286/AF, 03288/CU, CK. Viti Levu Island: S. \& T. Pócs 03252/BK, 03256/O, 03258/E, 03261/BM, $B N$.

Cololejeunea cordiflora is a new report for the islands of Taveuni and Viti Levu. The species is possibly new to Fiji Islands, although probably all previous records of C. goebelii refer to this species, see comments by Pócs \& Piippo (2011). 
59. Cololejeunea haskarliana (Lehm. \& Lindenb.) Schiffn., Conspectus Hepaticarum Archipelagi Indici: 244 (1898)

Basionym: Lejeunea haskarliana Lehm. \& Lindenb. Novarum et Minus Cognitarum Stirpium Pugillus 8: 26. 1844.

Type citation: Java, "Java insula, ubi in aliis Jumngermanniis repentem legit cl. Haskarl”.

\section{Cololejeunea haskarliana var. haskarliana}

Illustration: Benedix (1953: 134, t.18, 19a-b); Zhu \& So (2001: 323, fig. 121).

Specimens examined: Taveuni Island: S. \& T. Pócs 03279/BD, BE, BY.

Cololejeunea haskarliana var. haskarliana is a new report for the island of Taveuni. Cololejeunea haskarliana is distributed from the Seychelles and India to Japan, Australia and to New Caledonia (Pócs \& Streimann 2006).

60. Cololejeunea obliqua (Nees et Mont.) Schiffn., Botanische Jahrbücher für Systematik, Pflanzengeschichte und Pflanzengeographie 23: 586 (1897)

Basionym: Lejeunea obliqua Nees et Mont., Annales des Sciences Naturelles; Botanique, sér. 2 19: 264 (1843)

Type citation: French Guyana, "[cum L. oxyphylla] in foliis circa Cayennam á cl. Leprieur lecta”.

Illustration: Tixier (1985: 293, fig. 57, as C. scabriflora).

Species examined: Taveuni Island: 03279/AX, 03288/BNB, BC.

Cololejeunea obliqua is a new report for the island of Taveuni. This is a widespread pantropical species (Zhu \& So 2001).

61. Colura acroloba (Mont. ex Steph.) Ast, Revue Bryologique et Lichénologique 22: 297 (1953)

Basionym: Lejeunea acroloba Mont. ex Steph., Hedwigia 29: 97 (1890)

Type citation: Philippines [Luzon]. "Manila". Collector unknown. Holotype: W (Lindenb. Hepat. 6872) Lindenberg. (Zhu \& So 2001).

Illustration: Zhu \& So (2001: 230, fig. 88).

Specimens examined: Viti Levu Island: S. \& T. Pócs 03261,BK, BO.

Colura acroloba is a new report for the island of Viti Levu. Piippo (1994) noted the taxon as an Asian-Oceanian-Australian species.

62. Diplasiolejeunea cavifolia Steph., Species Hepaticarum 5: 918 (1916)

Basionym: Lejeunea cavifolia Steph., Botanische Jahrbücher für Systematik, Pflanzengeschichte und Pflanzengeographie 8: 89. 1886 [1887]

Type citation: Africa. Occid. Insula St. Thomé, Mt Caffe, 700 m, Sept. 1885, A. Moller 7a. (Zhu \& So 2001).

Illustration: Mizutani (1973: 198, fig. IV; 1973: 200, fig. V, as H. riddleana). 
Specimens examined: Taveuni Island: S. \& T. Pócs 03288/BK.

Diplasiolejeunea cavifolia is a new report for the island of Taveuni. Piippo (1994) noted the taxon was a transpacific species.

63. Harpalejeunea filicuspis (Steph.) Mizut., Journal of the Hattori Botanical Laboratory 37: 197 (1973)

Basionym: Drepanolejeunea filicuspis Steph., Species Hepaticarum 5: 344 (1913)

Type citation: Papua New Guinea, "Nova Guinea, Samarai”.

Illustration: Piippo (1985: 139, fig. 5 a-e).

Specimens examined: Kadavu Island: S. \& T. Pócs 03303/AR, 03304/A. Taveuni Island: S. \& T. Pócs 03289/BK pp. Viti Levu Island: T Pócs 08032/N.

Harpalejeunea filicuspis is a new report for the islands of Kadavu, Taveuni, and Viti Levu. Piippo (1994) noted the taxon was an Asian-Oceanian-Australian species.

64. Lejeunea discreta Lindenb., Synopsis Hepaticarum 3: 361 (1845)

Type citation: Indonesia. Java, “inter L. thymifoliam $\beta$ discretam (hb. N[ees])".

Illustration: Mizutani (1970: 237, fig. VII).

Specimens examined: Taveuni Island: S. \& T. Pócs 03289/CA.

Lejeunea discreta is a new report for the island of Taveuni; L. discreta is a widely distributed Indomalesian species (Pócs et al. 2007).

65. Lejeunea flava (Sw.) Nees, Naturgeschichte der Europäischen Lebermoose 3: 277 (1838)

Basionym: Jungermannia flava Sw., Nova Genera et Species Plantarum: 144 (1788)

Type citation: Jamaica. Swartz s.n. (Lectotype designated by Grolle 1976: UPSThunb.-25916, Isolectotype: BM, S).

Illustration: Schuster (1980: 959, fig. 689).

Specimens examined: Taveuni Island: S. \& T. Pócs 03279/BZ.

Lejeunea flava is a new report for the island of Taveuni. Lejeunea flava is a widespread pantropical species (Eggers 2004).

66. Lejeunea micholitzii Mizut., Journal of the Hattori Botanical Laboratory 33: 236 (1970)

nom. nov. pro Hygrolejeunea parvisaccata Steph., Species Hepaticarum 5: 567. 1914

Type citation: Luzon Benguet, 1884 Micholitz s.n. (Holotype: G-24700).

Illustration: Mizutani (1970: 237, fig. VI).

Specimens examined: Kadavu Island: S. \& T. Pócs 03309/L. Taveuni Island: S. \& T. Pócs 03279/ $B F, 03282 / G, 03288 / D C, 03289 / B V, B W$.

Lejeunea micholitzii is a new report for the islands of Kadavu and Taveuni. Recorded elsewhere from Sri Lanka, Vietnam, Borneo, Java, Philippines, New Caledonia, New Guinea Vanuatu and Easter Is. (cf. ELPT database). 
67. Lejeunea sordida (Nees) Nees, Naturgeschichte der Europäischen Lebermoose 3: 278 (1838)

Basionym: Jungermannia sordida Nees, Enumeratio Plantarum Cryptogamicarum Javae: 41 (1830)

Type citation: Indonesia. Java, "Inter muscos et Jungermanniae terrestres sparsim; in tumulis Baduorum sanctis; inter alias Jungermannias caespitosa, haud spernenda copia”.

Illustration: Mizutani (1970: 243, fig. XII).

Specimens examined: Kadavu Island: S. \& T. Pócs 03300/AP, 03304/BA, 03305/F, 03306/J. Taveuni Island: S. \& T. Pócs 03282/U.

Lejeunea sordida is a new report for the islands of Kadavu and Taveuni. Recorded elsewhere from Nicobar Is., Thailand, Borneo, Java, Moluccas, the Philippines, Sulawesi, Bismarck Archipelago, New Guinea, Solomon Is., Queensland, New Caledonia, Samoa, Caroline Is., and the Marianas (cf. ELPT database).

68. Lejeunea umbilicata (Nees) Nees, Novorum Actorum Academiae Caesareae Leopoldino-Carolinae Naturae Curiosorum 19 suppl. 1: 472 (1843)

Basionym: Jungermannia umbilicata Nees, Enumeratio plantarum cryptogamicarum Javae: 42. (1830)

Type citation: Java, "Inter muscos Javae frequens, saepe fructifera invenitur".

Illustration: Mizutani (1970: 23, fig. V).

Specimens examined: Taveuni Island: S. \& T. Pócs 03278/O, V, 03279/H, AG, 03286/L.

Lejeunea umbilicata is a new report for the island Taveuni. This species has a Southeast Asian and Pacific distribution, including Borneo, Java, Philippines, Sumatra, New Guinea, New Caledonia, Samoa, Caroline Is., and the Marianas (cf. ELPT database).

69. Lepicolea rara (Steph.) Grolle, Nova Hedwigia 16: 152 (1968)

Basionym: Lepidozia rara Steph., Species Hepaticarum 3: 618 (1909)

Type citation: Indonesia. "Java (leg.?)".

Illustration: Piippo (1984: 23, fig. 2).

Specimens examined: Taveuni Island: S. \& T. Pócs 03279/S.

Lepicolea rara is a new report for the island of Taveuni. It has been recorded elsewhere from Thailand, Malay Peninsula, Sumatra, Borneo, Philippines, Sulawesi, Moluccas, Java, New Guinea, Solomon Islands, Society Is, and Chile (cf. ELPT database).

70. Lepidolejeunea bidentula (Steph.) R.M.Schust., Phytologia 45: 425 (1980)

Basionym: Lejeunea bidentula Steph., Handel-Mazetti, Symbolae Sinicae 5: 51 (1930)

Type citation: Papua New Guinea "Fly River branch, leg. W. Bäuerlen, 1885”. 
Illustration: Piippo (1986: 22-23, figs 4-5).

Specimens examined: Kadavu Island: S. \& T. Pócs 03301/G, 03307/C. Taveuni Island: S. \& T. Pócs 03288/Z.

Lepidolejeunea bidentula is a new report for the islands of Kadavu, Taveuni, and Viti Levu. This species has a paleotropical distribution (Piippo 1994).

71. Lepidolejeunea graeffei (J.B.Jack et Steph.) R.M.Schust., Phytologia 45: 425 (1980)

Basionym: Archilejeunea graeffei J.B.Jack et Steph., Botanisches Centralblatt 60: 104 (1894)

Type citation: Fiji. Viti-Ovalau, in summo monte Tana-lailai, 1864 Graeffe (Lectotype designated by Piippo 1986: G-22718 c. per., provisionally selected by Grolle 1983 in sched., isolectotypes: BM, G-14014, G-14015) fide Piippo 1986b.

Illustration: Piippo (1986: 31, fig. 8).

Specimens examined: Taveuni Island: S. \& T. Pócs 03288/BT.

Lepidolejeunea graeffei is a new report for the island of Taveuni. Piippo (1994) reports this taxon as an Asian-Oceanian species; with records from Philippines, New Guinea, Bismarck Archipelago, Solomon Islands, Vanuatu, Fiji, Samoa, and Caroline Is (cf. ELPT database).

72. Lepidolejeunea integristipula (J.B.Jack et Steph.) R.M.Schust., Phytologia 45: 425 (1980)

Basionym: Pycnolejeunea integristipula J.B.Jack et Steph., Botanisches Centralblatt 60: 107 (1894)

Type citation: Fiji, "Viti-Ovalau, in monte Tana-lailai. Pauca specimina Taxi-Lejeuneam umbilicatum perrepentia".

Illustration: Piippo (1986: 35, fig. 9).

Specimens examined: Kadavu Island: $S$. \& T. Pócs 03300/S, 03303/K, 03305/B, 03307/AC. Taveuni Island: S. \& T. Pócs 03283/Q, 03289/BU.

Lepidolejeunea integristipula is a new report for the islands of Kadavu and Taveuni. Piippo (1994) reports this taxon as an Asian-Oceanian species; with records from Borneo, Philippines, Java, New Guinea, Solomon Islands, Queensland, Fiji, Samoa, and Caroline Is (cf. ELPT database).

73. Lopholejeunea eulopha (Taylor) Schiffn., Nat. Pflanzenfam. [Engler \& Prantl]: 129 (1893)

Basionym: Lejeunea eulopha Taylor, London Journal of Botany 5: 391 (1846)

Type citation: Pacific Is., Nightingale s.n. Holotype: FH; Isotype: NY. (Gradstein et al. 2002).

Illustration: Thiers \& Gradstein (1989: 26, fig. 8).

Specimens examined: Kadavu Island: S. \& T. Pócs 03304/BB. Taveuni Island: S. \& T. Pócs 0303279/P. Det. A. Sass-Gyarmati. 
Lopholejeunea eulopha is a new report for the island of Kadavu. The species is Palaeotropic, widely distributed in Asia and Africa, and rather frequent in Queensland (Australia) (Sass-Gyarmati 2002).

74. Lopholejeunea nigricans (Lindenb.) Schiffn., Conspectus Hepaticarum Archipelagi Indici: 293 (1898)

Basionym: Lejeunea nigricans Lindenb., Synopsis Hepaticarum 3: 316 (1845)

Type citation: Indonesia. Java, unknown coll. Holotype: W-Lindenb. (Gradstein et al. 2002); Isotype: G (Gradstein et al. 2002; Zhu \& Gradstein 2005).

Illustration: Gradstein et al. (2002: 34, fig.19).

Specimens examined: Kadavu Island: S. \& T. Pócs 03330/AS. Taveuni Island: S. \& T. Pócs 0303278/J, 03285/M, 03288/AE, 03293/Y, 03299/M, T. Pócs 08012/AB. Det. A. Sass-Gyarmati.

Lopholejeunea nigricans is a new report for the islands of Kadavu and Taveuni. This species has a pantropical distribution (Piippo 1994).

75. Lopholejeunea subfusca (Nees) Schiffn., Botanische Jahrbücher für Systematik, Pflanzengeschichte und Pflanzengeographie 23: 593 (1897)

Basionym: Jungermannia subfusca Nees, Enumeratio Plantarum Cryptogamicarum Javae: 36 (1830)

Type citation: Indonesia. Java, Blume s.n. Holotype: W; Isotype S-B29157 (Gradstein et al. 2002; Zhu \& Gradstein 2005).

Illustration: Thiers \& Gradstein (1989: 40, fig. 15).

Specimens examined: Kadavu Island: S. \& T. Pócs 03301/F, 03310/C. Taveuni Island: S. \& T. Pócs 03279/P, 03282/AG, 03285/B, 03279/P, 03282/AG, 03285/B. Det. A. Sass-Gyarmati.

Lopholejeunea subfusca is a new report for the islands of Kadavu and Taveuni. This is a widespread pantropical species (Sass-Gyarmati 2002).

76. Mastigolejeunea calcarata (Steph.) Verd., Blumea 1:218, 230 (1934)

Basionym: Archilejeunea calcarata Steph., Species Hepaticarum 4: 724 (1911)

Type citation: Fiji. "Viti, Ovalau (Seemann)": NY sub Phragmicoma calcarata Mitt.

Illustration: Thiers \& Gradstein (1989: 45, fig. 16).

Specimens examined: Kadavu Island: S. \& T. Pócs 03304/M, 03305/K, 03309/F. Det. A. SassGyarmati.

Mastigolejeunea calcarata is a new report for the island of Kadavu. Thiers \& Gradstein (1989) also reported this taxon from Australia.

77. Metalejeunea crassitexta (J.B.Jack et Steph.) Pócs, comb. nov.

Basionym: Microlejeunea crassitexta J.B.Jack et Steph., Botanisches Centralblatt 60: 106 (1894)

Type citation: Fiji. "Viti-Ovalau in monte Tana-Lailai", 1864, leg. Graeffe.

This species, purportedly an endemic to the Fiji Islands, was previously known 
only from the sterile type material. It shares many characters with Metalejeunea cucullata (Reinw. et al.) Grolle. We have found on the specimen from Kadavu Island well-developed perichaetia with Pycnolejeunea-type subgynoecial innovations. The pycnolejeuneoid innovation is a critical character of Metalejeunea that distinguishes it from Microlejeunea which has lejeuneoid innovations. Thus, we hereby transfer Microlejeunea crassitexta J.B.Jack et Steph into Metalejeunea. In addition, this taxon has no basal ocelli, which is a character of Metalejeunea, in contrast to Microlejeunea, where basal ocelli regularly occur. Metalejeunea winkleri Zhu \& Grolle (2002), another species with small lobe cells might be conspecific, if the Fijian taxon has flat perianths.

Illustration: Miller, Bonner \& Bischler (1963: 4, tab. 131, fig. a-e, as Microlejeunea crassitexta).

Specimens examined: Taveuni Island: S. \& T. Pócs 03288/DR. Kadavu Island: S. \& T. Pócs 03303/ AS.

Metalejeunea crassitexta is a new report for the islands of Taveuni and Kadavu. It is previously known from Ovalau and Viti Levu (Jack \& Stephani 1894).

78. Metalejeunea cucullata (Reinw., Blume et Nees) Grolle, Bryophytorum Bibliotheca 48: 100 (1995)

Basionym: Jungermannia cucullata Reinw., Blume et Nees, Nova Acta PhysicoMedica Academiae Caesareae Leopoldino-Carolinae Naturae Curiosorum Exhibentia Ephemerides sive Observationes Historias et Experimenta 12: 227 (1825)

Type citation: Indonesia. Java, "in lava insula ad terram. Blume. V. c. fr.".

Illustration: Zhu \& So (2001: 167, fig. 65).

Specimens examined: Kadavu Island: S. \& T. Pócs 03307/N.

Metalejeunea cucullata is a new report for the island of Kadavu. Metalejeunea cucullata is a pantropical species (Zhu \& Grolle 2002).

79. Metzgeria furcata (L.) Nees, Naturgeschichte der Europäischen Lebermoose 3: 485. 1838)

Basionym: Jungermannia furcata L., Species Plantarum: 1136 (1753)

Type citation: Lectotype (Grolle \& So 2002): [icon.] "Lichenastrum tenuifolium, furcatum, thecis globosis pilosis" in Dillenius, Historia Muscorum t. 74, f. 45 A-C, F-G (excl. D-E, see Lindberg 1878: 32, 36). 1742 "1741". Epitype (Grolle \& So 2002): OXF (hb. Dillenius f.)

Illustration: Schuster (1992: 680, fig. 886).

Specimens examined: Taveuni Island: S. \& T. Pócs 03288/D, AC.

Metzgeria furcata is a new report for the island of Taveuni. The treatment of Australasian and Pacific Metzgeria by So (2002) was followed in this determination. However, it is clear that $M$. furcata represents a group with a complex taxonomy. Recently, Fuselier et al. (2009), in a molecular phylogeny of Northern Hemisphere accessions concluded that M. furcata, has a wide disjunct distribution, with populations exhibiting extreme sex ratios, and harbours cryptic lineages. 
80. Phaeolejeunea amicorum (Hürl.) Pócs, Fieldiana: Botany, n.s. 47: 140 (2008)

Basionym: Phaeolejeunea etesseana subsp. amicorum Hürl., Bauhinia 9: 263 (1991)

Type citation: Tonga, "mit anderen Lejeuneaceen auf Rinde eines Baums in mesophilen Primärwald, am Weg von Fuai zum Eastrern Ridge (,Eua)”, ca 130 m ü M. 9. XI. 1951, leg. H.H. T837 (Holotype: Z, isotypes: G. hb. Hürlimann).

Illustration: Pócs (2008a: 141-142, figs 1-2).

Specimens examined: Viti Levu Island: T. Pócs 03275/G, 08032/B.

Phaeolejeunea amicorum is a new report for the island of Viti Levu. This species had previously been recorded in Fiji (Taveuni and Kadavu) by Pócs (2008a). Prior to that it was only known from Tonga (Hürlimann 1991).

81. Plagiochilion oppositum (Reinw., Blume et Nees) S.Hatt., Biosphaera 1: 7 (1947)

Basionym: Jungermannia opposita Reinw., Blume et Nees, Nova Acta Physico-Medica Academiae Caesareae Leopoldino-Carolinae Naturae Curiosorum Exhibentia Ephemerides sive Observationes Historias et Experimenta 12: 236 (1825)

Type citation: Indonesia. Java, "Inter Hypna et Iungermannias, v. .c. I. scorpioidem, Iavae insulae invenit Blume. V. s. fr.”.

Illustration: Inoue (1964: 62, fig. VI).

Specimens examined: Viti Levu Island: T. Pócs 08023/A.

Plagiochilion oppositum is a new report for the island of Viti Levu. It is recorded elsewhere from Guangxi, Hainan, Japan, Taiwan, India, Sri Lanka, Burma (Myanmar), Thailand, Vietnam, Borneo, Java, Lesser Sunda Is., Philippines, Sulawesi, Sumatra, New Guinea, Solomons, Queensland, New Caledonia, Samoa, and Vanuatu (cf. ELPT database).

82. Ptychanthus striatus (Lehm. et Lindenb.) Nees, Naturgeschichte der Europäischen Lebermoose 3: 212 (1838)

Basionym: Jungermannia striata Lehm. et Lindenb., Novarum et Minus Cognitarum Stirpium Pugillus 4: 16 (1832)

Type citation: Nepal. "in Nepalia”.

Illustration: Thiers \& Gradstein (1989: 56, fig. 21); Gradstein et al. (2002: 57, fig. 35).

Specimens examined: Taveuni Island: S. \& T. Pócs 03288/O. Det. A. Sass-Gyarmati.

Ptychanthus striatus is a new report for the island of Taveuni. This species has a paleotropical distribution (Piippo 1994).

83. Radula acuminata Steph., Species Hepaticarum 4: 230 (1910)

Type citation: Vietnam. “Tonkin”, 18 Oct 1887, B. Balansa s.n., herb. Paris 1890. Holotype: G-35916; Isotype: G-35915, PC (So 2006).

Illustration: Yamada (1979: 287, fig. 47).

Specimens examined: Taveuni Island: S. \& T. Pócs 0379/BA.

Radula acuminata is a new report for the island of Taveuni. The species has a wide 
distribution including China, Japan, Sri Lanka, Cambodia, Thailand, Vietnam, Borneo, Java, New Guinea and New Caledonia (cf. ELPT database).

84. Radula multiflora Gottsche ex Schiffn., Forschungsreise S.M.S. 'Gazelle'. 4, Botanik: 20 (1889) [1890]

Type citation: New Guinea. Bismarck Archip., New Hannover Island, 20 Jul 1875, Gazelle Exped., F. C. Natonann s.n. as R. javanica (Lectotype designated by Castle 1966: FH-60323, isolectotypes: W-5145, YU). New Ireland. ins. '... Neu-Mecklenburg..' 19 Aug 1875, F. C. Na.

Illustration: Renner \& de Lange (2009: 3, fig. 1, 2).

Specimens examined: Taveuni Island: S. \& T. Pócs 03288/AH, 03293/A, Z, 03294/B, 03295/F, 03298/T. Kadavu Island: S. \& T. Pócs 03303/X.

Radula multiflora is a new report for the islands of Taveuni and Kadavu. This species is found throughout SE Asia and the western pacific, from Sumatra to New Caledonia, Samoa and Tahiti (Yamada 1979).

85. Radula protensa Lindenb., Botanische Zeitung. Berlin 6: 462 (1848)

Type citation: Indonesia. Java. Zollinger 577. Holotype: BM; Isotype: G, P (Zhu \& So 2001).

Illustration: Yamada (1979: 293, fig. 51).

Specimens examined: Taveuni Island: S. \& T. Pócs 03286/AB.

Radula protensa is a new report for the island of Taveuni. It has been recorded elsewhere from India, Sumatra, Borneo, Philippines, Java, New Guinea, and Society Is (cf. ELPT database).

86. Spruceanthus polymorphus (Sande Lac.) Verd., Annales Bryologici, suppl. 4: 155 (1934)

Basionym: Phragmicoma polymorpha Sande Lac., Nederlandsch Kruidkundig Archief. Verslangen en Mededelingen der Nederlandsche Botanische Vereeniging 3: 420 (1854) [1855]

Type citation: Indonesia. Java, leg. Junghuhn. Source: IH online.

Illustration: Gradstein et al. (2002: 69, fig. 43).

Specimens examined: Kadavu Island: S. \& T. Pócs 03300/AO. Det. A. Sass-Gyarmati.

Spruceanthus polymorphus is a new report for the island of Kadavu. This species has a broad distribution throughout southeast Asia and the Pacific, including China, Japan, India, Indonesia, New Guinea, Philippines, Australia, and islands throughout the South Pacific (Zhu \& So 1999). Gradstein et al. (2002) discussed the relationship between Spruceanthus and Archilejeunea, where some authors have placed it, yet they retained the species under Spruceanthus until the oil bodies could be studied on living material. This will be imperative in future collections in Fiji. 
87. Thysananthus spathulistipus (Reinw., Blume et Nees) Lindenb., Synopsis Hepaticarum 2: 287 (1845)

Basionym: Jungermannia spathulistipa Reinw., Blume et Nees, Nova Acta PhysicoMedica Academiae Caesareae Leopoldino-Carolinae Naturae Curiosorum Exhibentia Ephemerides sive Observationes Historias et Experimenta 12: 212 (1825)

Type citation: Indonesia. Java, "in montibus (v. c. Monte Leback) Provinciae Bantam Iavae insula. Blume. V. c. cal.”.

Illustration: Thiers \& Gradstein (1989: 74, fig. 28); Gradstein et al. (2002: 81, fig. 51).

Specimens examined: Kadavu Island: S. \& T. Pócs 03304/M. Det. A. Sass-Gyarmati.

Thysananthus spathulistipus is a new report for the island of Kadavu. This is a pantropical species widely distributed in Africa, Southeast Asia and Oceania (Sass-Gyarmati 2003).

\section{Acknowledgments}

The financial assistance of the National Geographic Committee for Research and Exploration (Grant No. 8247-07), GBIF Seed Money Award No. 2007/41, and the Field Museum Woman's Board, Field of Dreams Program are warmly acknowledged. The first author was financed in 2003 by the AMFK (Hungarian Ministry of Education) and by ALCOA Foundations (USA). He is also thankful for his wife, Sarolta Pócs's field assistance. The authors also are especially grateful to Anders Hagborg and Lars Söderström of the Early Land Plants Today (ELPT) project for their comments and corrections as well as access to their databases. We also express our gratitude to Matt Renner for some useful comments as well John J. Engel and Endymion Cooper for useful discussion on Telaranea. We warmly thank the valuable assistance, guidance, and logistical support provided by the Suva Herbarium, support in the field by Jone Navuso.

\section{References}

Allorge V \& Jovet-Ast S (1950) Aphanolejeunea teotonii nov. sp., hépatique des Açores Revue Bryologique et Lichénologique 19: 19-24.

Asthana G. \& Srivastava SC (2003) Indian Cololejeunea. A taxonomic study. Bryophytorum Bibliotheca 60: 1-155.

Benedix EH (1953) Indomalayische Cololejeuneen Feddes Repertorium Specierum Novarum Regni Vegetabilis. Beiheft 134: 1-88.

Bonner CEB (1953) De Hepaticis III. A contribution to the study of the genus Ceratolejeunea (Spruce) Schiffner. Candollea 14: 163-252

Braggins JE (1999) Cololejeunea cardiocarpa (Lejeuneacee), a new hepatic record in New Zealand. New Zealand Journal of Botany 37: 79-82.

Daniels AED, Kariyappa KC \& DanielP (2010) Circumscription of the polymorphic Cololejeunea lanciloba Steph.(Lejeuneaceae, Hepaticae) and species falling within it. Acta Botanica Hungarica 52: 287-295.

DoE (Department of the Environment) (1997) Convention on Biological Diversity 1997. National Report to the Conference of the Parties by the Republic of Fiji. (Department of the Environment, Ministry of Local Government, Housing and Environment: Suva, Fiji).

Eggers J (2004) New bryopyte taxon records for tropical countries 5. Tropical Bryology 25: 19-23. 
Eggers J, Frahm JP \& Pursell RA (1998) New bryophyte taxon records for tropical countries II. Tropical Bryology 14: 81-84.

Eifrig H (1937) "1936": Monographische studien über die. Indomalayischen Arten von Taxilejeunea. Annales Bryologici 9: 73-114.

Engel JJ (2006) Austral Hepaticae 42. The Austral species of Mnioloma (Calypogeiaceae), together with a new species, Mnioloma novaezelandiae n. sp. Cryptogamie Bryologie 27: 111-117.

Engel JJ \& Smith Merrill GL (2004) Austral Hepaticae 35. A taxonomic and phylogenetic study of Telaranea (Lepidoziaceae), with a monograph of the genus in temperate Australasia and commentary on extra-Australasian taxa. Fieldiana, Botany, n.s. 44: 1-265.

Engel JJ \& Glenny D (2008) A Flora of the Liverworts and Hornworts of New Zealand. vol. 1. St. Louis, Missouri Botanical Garden Press, 897 pp.

Evans AW (1905) New or noteworthy Hepaticae from Florida. Bulletin of the Torrey Botanical Club 32: 179-192.

Fuselier L, Davison PG, Clements M, Shaw B et al. (2009) Phylogeographic analyses reveal distinct lineages of the liverworts Metzgeria furcata (L.) Dumort. and Metzgeria conjugata Lindb. (Metzgeriaceae) in Europe and North America, Biological Journal of the Linnean Society 98: 745-756.

Gradstein SR, He-Nygrén XL, Piippo S \& Mizutani M (2002) Bryophyte flora of the Huon Peninsula, Papua New Guinea. LXVIII. Lejeuneaceae subfamily Ptychanthoideae (Hepaticae). Acta Bot. Fennica 174: 1-88.

Grolle R (1976) Verzeichnis der Lebermoose Europas und benachbarter Gebiete. Feddes Repertorium 87: 171-279.

Grolle R (1979) Miscellania Hepaticologica 181-190. Journal of the Hattori Botanical Laboratory 45: 173-183.

Grolle R (1980) Zur kenntnis der Lebermoose von Samoa I. Wissenschaftliche Zeitschrift der Friedrich-Schiller-Universität Jena/Thüringen. Matematisch-naturwissenschaftliche Reihe 29: 637-648.

Grolle R \& So ML (2002) Typifications of three old names of Metzgeria species (Hepaticae): Jungermannia furcata L. $1753 \mathrm{~m}$ J. linearis Sw. 1788 and J. pubescens Schrank 1792. Cryptogamie, Bryologie 23: 119-121.

Herzog T (1932) Neue Hepaticae aus der weiteren Indomalaya. Annales Bryologici 5: 83-98.

Herzog T (1936) Studien über Drepanolejeunea III. Annales Bryologici 9: 115-130.

Hürlimann H (1987) Hepaticae aus dem Gebiete des südlichen Pazifik IX. Bauhinia 8: 221-234.

Hürlimann H (1991) Hepaticae aus dem Gebiete des südlichen Pazifik. XI. Bauhinia 9: 257-264.

Inoue H (1964) The genus Plagiochilion. Journal of the Hattori Botanical Laboratory 27: 51-72.

Isono S (2009) Lejeuneaceae in Kanagawa, Japan 2. Natural Environmental Science Research 22: $39-50$.

Jack JB \& Stephani F (1894) Hepaticae in insulis Vitibuensibus et Samoanis a Dre Ed. Graeffe anno 1864 lectae. Botanisches Centralblatt 60: 97-109.

Jones EW (1972) African hepatics XXIII. Some species of Lejeunea. Journal of Bryology 7: 23-45.

Jovet-Ast S (1953) Le genre Colura: Hépatiques, Lejeuneaceae, Diplasieae. Revue Bryologique et Lichénologique, Nouvelle Série 22: 206-312.

Kachroo P \& Schuster RM (1961) The genus Pycnolejeunea and its affinities to Cheilolejeunea, Euosmolejeunea, Nipponolejeunea, Tuyamaella, siphonolejeunea and Strepsilejeunea. Journal of the Linnean Society. Botany 56: 475-511.

Miller HA (1956) Cryptogams of Kapingamarangi Atoll, Carolina Islands. I. Bryophyta. The Bryologist 59: 167-173.

Miller HA (1968) Bryophyta of Guam and northern Micronesia. Micronesica 4: 49-83.

Miller HA (1986) Pacific Bryophytes 3. An overview of Telaranea, Hepatophyta. Journal of Bryology 14: 231-244.

Miller HA, Whittier HO \& Bonner CEB (1963a) Bryoflora of the atolls of Micronesia. Beihefte zur Nova Hedwigia 11: 1-92.

Miller, H.A., Bonner, CEB. and Bischler H (1963b) Studies in Lejeuneaceae V. Microlejeunea in Pacific Oceania. Nova Hedwigia 4: 551-560. 
Miller HA, Whittier HO \& Whittier BA (1983) Prodromus florae hepaticarum Polynesiae. Catalogue of Hepaticae and Anthocerotae. Bryophytorum Bibliotheca 25: 1-423.

Mizutani M (1961) A revision of Japanese Lejeuneaceae. Journal of the Hattori Botanical Laboratory 24: 115-302.

Mizutani M (1963) On some Indian species of the family Lejeuneaceae described by W. Mitten. Journal of the Hattori Botanical Laboratory 26: 171-184.

Mizutani M (1965) Studies of little known Asiatic species of Hepaticae in the Stephani Herbarium. 2. On some little known southeast Asiatic species of the genus Cololejeunea. Journal of the Hattori Botanical Laboratory 28: 107-121.

Mizutani M (1967) Studies of little known asiatic species of Hepaticae in the Stephani Herbarium. 3. On some little known species of Cheilolejeunea, Euosmolejeunea and Pycnolejeunea. Journal of the Hattori Botanical Laboratory 30: 171-180.

Mizutani M (1968) Studies of little known Asiatic species of Hepaticae in the Stephani Herbarium. 4. Phaeolejeunea, a new genus of Lejeuneaceae. Journal of the Hattori Botanical Laboratory 31: 130-134.

Mizutani M (1970) Lejeuneaceae, subfamilies Lejeuneoideae and Cololejeuneoideae, from Sabah (North Borneo). Journal of the Hattori Botanical Laboratory 33: 225-265.

Mizutani M (1973) The genus Harpalejeunea from Sabah (North Borneo). Journal of the Hattori Botanical Laboratory 37: 191-203.

Mizutani M (1980) Notes on the Lejeuneaceae. 3. Some Asiatic species of the genus Cheilolejeunea. Journal of the Hattori Botanical Laboratory 47: 319-331.

Mizutani M (1982) Notes on the Lejeuneaceae. 6. Japanese species of the genus Cheilolejeunea. Journal of the Hattori Botanical Laboratory 51: 151-173.

Muzutani M (1990) Notes on the Lejeuneaceae. 16. Drepanolejeunea thwaitesiana and its related species from Asia. Journal of the Hattori Botanical Laboratory 68: 367-380.

Piippo S (1984) Bryophyte flora of the Huon Peninsula, Papua New Guinea. III. Haplomitriaceae, Lepicoleaceae, Herbertaceae, Pseudolepicoleaceae, Trichocoleaceae, Schistochilaceae, Balantiopsaceae, Pleuroziaceae and Porellaceae (Hepaticae). Annales Botanci Fennici 21: $21-48$.

Piippo S (1985) Bryophyte flora of the Huon Peninsula, Papua New Guinea. XII. Geocalycaceae (Hepaticae). Acta Botanica Fennica 131: 129-167.

Piippo S (1986) A monograph of the Lepidolejeunea and Luteolejeunea (Lejeuneaceae: Hepaticae). Acta Botanica Fennica 132: 1-69.

Piippo S (1994) On the bryogeography of Western Melanesian Lejeuneaceae, with comments on their epiphyllous occurrence. Tropical Bryology 9: 43-58.

Pócs T (2008a) Bryophytes from the Fiji Islands, III. The genus Phaeolejeunea Mizut. (Lejeuneaceae), with detailed description of P. amicorum (Hürl.) Pócs, stat. nov. Fieldiana: Botany, n.s. 47: 139-145.

Pócs T (2008b) Bryophytes from the Fiji Islands, IV. The genus Frullania Raddi (Jungermanniopsida), I., with description of F. vivipara Pócs, spec. nov. Fieldiana, Botany 47: $147-158$.

Pócs T (2010) On some less known Lejeunea (Lejeuneaceae, Jungermanniopsida) species in tropical Africa. East African Bryophytes XXVII (with 9 plates). Nova Hedwigia, Beiheft 138: 99-116.

Pócs T. \& Eggers J (2007) Bryophytes from the Fiji Islands, II. An account of the genus Colura, with the description of C. vitiensis sp. nov. Polish Botanical Journal 52(2): 81-92.

Pócs T \& Streimann H (2006) Contributions to the bryoflora of Australia I Tropical Bryology 27: 19-24.

Pócs T \& Bernecker A (2009) Overview of Aphanolejeunea (Jungermanniopsida) after 25 years. Polish Botanical Journal 54: 1-11.

Pócs T \& Piippo S (2011) Bryophyte flora of the Huon Peninsula, Papua New Guinea. LXXI. Cololejeunea (Lejeuneaceae, Jungermanniopsida). Acta Bryolichenologica Asiatica 4: (in press). 
Pócs, T, Nair MC, Rajesh KP \& Madhusoodanan PV (2007) Liverwort (Marchantiopsida) records from the Western Ghats (Kerala State, Peninsular India). Acta Botanica Hungarica 49: 121-129.

Renner MAM \& Braggins JE (2006) Hepatic records for Australia and New Zealand. Australasian Bryological Newsletter 52: 9-13.

Renner MAM \& Brown EA (2008) Mnioloma (Calypogeiaceae: Jungermanniopsida) in Australasia: how many species are there? Fieldiana, Botany 47: 159-174.

Renner MAM \& de Lange PJ (2009) Radula multiflora Gottsche ex Schiffn. - a new record for the Chatham Islands, New Zealand. Australasian Bryological Newsletter 57: 12-13.

Sass-Gyarmati A (2002) Ptychanthoideae (subfam. of Lejeuneaceae, Hepaticae) from Vanuatu, with the description of Caudalejeunea streimannii Gyarmati sp. n. Tropical Bryology 22: $125-134$.

Sass-Gyarmati A (2003) Records on the distribution of Ptychanthoideae (subfam. of Lejeuneaceae, Hepaticae) in Australia. Tropical Bryology 24: 21-31.

Schuster RM (1980) The Hepaticae and Anthocerotae of North America IV. (New York: Columbia University Press) $133 \mathrm{pp}$. not sure if the pp are enclosed in the parentheses

Schuster RM (1992) The Hepaticae and Anthocerotae of North America VI. (New York: Columbia University Press) 937 pp.

Schuster RM (2000) Austral Hepaticae, Part. 1. Nova Hedwigia, Beiheft 118: 1-524.

So ML (2000) Studies on Plagiochila (Hepaticae) in Australasia and the Pacific II. Cryptogamie, Bryologie 21: 223-231.

So ML (2002) Metzgeria (Hepaticae) in Australasia and the Pacific. New Zealand Journal of Botany 40: 603-627.

So ML (2006) Radula (Radulaceae, Marchantiophyta) in the South Pacific. Journal of the Hattorial Botanical Laboratory. 99: 207-232.

Söderström L, Gradstein SR \& Hagborg A (2010) Checklist of hornworts and liverworts for Java. Phytotaxa 9: 53-149.

Staples GW \& Imada CT (2007) Checklist of Hawaiian Anthocerotes and Hepatics. Tropical Bryology 28: 15-47.

Stephani F (1985) Icones Hepaticarum, Jardin Botanique Genève, 12315 pp. IDC, Zug (165 microfiches).

Thiers BM (1988) The Australian species of Cololejeunea. Beihefte zur Nova Hedwigia 90: 113-146

Thiers BM (1993) A monograph of Pleurozia (Hepaticae; Pleuroziaceae). The Bryologist 96: 517-554.

Thiers BM \& Gradstein SR (1989) Lejeuneaceae (Hepaticae) of Australia. I. Subfamily Ptychanthoideae. Memoirs of the New York Botanical Garden 52: 1-79.

Tixier P (1979) Contribution à l'étude du genre Cololejeunea. Les Cololejeunoidées de Nouvelle Caledonie. Nova Hedwigia 31: 721-787.

Tixier P (1985) Contribution à la Connaissance des Cololejeunoideae. Bryophytorum Bibliotheca 27: $1-440$.

Tixier P (1993) Information on the Genus Cololejeunea (Lejeuneaceae, Hepaticae) XI. Some Pedinolejeunea in Austral Regions. Cryptogamie: Bryologie, Lichénologie 14: 353-360.

von Konrat M, Naikatini A, Tuiwawa M, Söderström L, Fife A, Renner M, Brownsey P, Perrie L, Hagborg A, Pócs T, Lumbsch HT, Braggins J, Séneca A \& Brown E (2011) A brief history of the cryptogams of Fiji and prospects for the future. Telopea 13:361-374.

Yamada K (1979) A revision of Asian taxa of Radula, Hepaticae. Journal of the Hattori Botanical Laboratory 45: 201-322.

Zhu RL (1995) Notes on some species of the genus Cololejeunea (Lejeuneaceae, Hepaticae) in China. Journal of the Hattori Botanical Laboratory 78: 83-109.

Zhu RL (2005) Cololejeunea aequabilis (Sande Lac.) Schiffn. (Jungermanniopsida: Lejeuneaceae), the correct name for Cololejeunea yulensis (Steph.) Benedix. Journal of Bryology 27:357-358. 
Zhu RL \& Gradstein SR (2005) Monograph of Lopholejeunea (Spruce) Schiffn. (Lejeuneaceae, Hepaticae) in Asia. Monographs in Systematic Botany from the Missouri Botanical Garden 74: 1-98.

Zhu RL \& Grolle R (2002) Metalejeunea winkleri R.-L. Zhu \& Grolle (Lejeuneaceae, Hepaticae), a new species from Borneo. Nova Hedwigia 74: 497-500.

Zhu RL \& Grolle R (2003) Taxonomy and distribution of Lejeunea exilis (Lejeuneaceae, Hepaticae). Annales Botanici Fennici 40: 101-106.

Zhu R-L \& Grolle R (2004) Nomenclatural notes on Cheilolejeunea inaequitexta and C. trapezia (Lejeuneaceae, Hepaticae). Annales Botanci Fennici 41: 445-447.

Zhu RL \& So ML (1999a) Additions to the Lejeuneaceae taxa to the hepatic flora of Yunnan, China. Annales Botanici Fennici 36: 219-229.

Zhu RL \& So ML (1999b) New records of Cololejeunea for China and Vietnam Botanical Bulletin of Academia Sinica, n.s. 40: 165-171.

Zhu RL \& So ML (2001) Epiphyllous liverworts of China. Beihefte zur Nova Hedwigia 121: 1-418.

Zhu RL \& So ML (2002) Reappraisal of Cololejeunea angustiflora, C. ciliata, C. flavicans, C. javanica and C. mackeeana (Hepaticae, Lejeuneaceae). The Bryologist 105: 431-438.

Zhu RL, Long DG \& Yamaguchi T (1999) Cololejeunea yulensis (Steph.) Benedix and Raphidolejeunea spicata (Steph.) Grolle new to Japan, with taxonomic notes on Cololejeunea obliqua (Nees \& Mont.) Schiffn. Journal of Bryology 21: 293-297.

Zhu RL, So ML \& Wang Y-F (2002) The genus Cheilolejeunea (Hepaticae, Lejeuneaceae) in China. Nova Hedwigia 75: 387-408.

Manuscript received 20 January 2011, accepted 22 August 2011

\section{Appendix 1: List of localities}

The abbreviated collection data provided in the specimen examined sections can be cross referenced below with the full detailed collection data.

03252: W part of VITI LEVU Island, at the N edge of NAUSORI Highlands, ENE of NAUSORI village, at 600-620 m alt. S 1749.033', E 177³7.338-38.046'. Secondary submontane rainforest with moderate number of epiphytes incl. epiphylls. 18. Aug. 2003.

03253: W part of VITI LEVU Island, at the N edge of NAUSORI Highlands, W of BUKUYA village, at $570 \mathrm{~m}$ alt. S $17^{\circ} 48.173^{\prime}$, E $127^{\circ} 39.675^{\prime}$. Submontane rainforest, streamside type with higher number of epiphytes. 18. Aug. 2003.

03255: Southern coast of VITI LEVU Island ('Coral Coast') in the high rainfall area. Tambua Sands Beach Resort E of NAMADA village, at 3-5 m alt. S 18 11.472', E 177³7.582'. Coconut stand and garden trees. 19. Aug. 2003.

03256: Southern coast of VITI LEVU Island ('Coral Coast') in the high rainfall area. $1.2 \mathrm{~km}$ N of KOROLEVU, at $34 \mathrm{~m}$ alt. S 18 $11.025^{\prime}$, E $177^{\circ} 41.914^{\prime}$. Lowland rainforest along streamside in a side valley, rich in epiphytes, incl. epiphylls. 19. Aug. 2003

03258: Southern coast of VITI LEVU Island ('Coral Coast') in the high rainfall area. In the narrow valley below BIASEVU ('Savu Na Mate Laya') waterfall, $2.5 \mathrm{~km} \mathrm{~N}$ of KOROLEVU. S $18^{\circ} 11-12^{\prime}$, E $177^{\circ} 42^{\prime}$. Wet lowland rainforest on volcanic ground. 19. Aug. 2003.

03259: Southern coast of VITI LEVU Island ('Coral Coast') in the high rainfall area. PACIFIC HARBOUR township area, at 2-3 $\mathrm{m}$ alt. S $18^{\circ} 15.041^{\prime}$, E $178^{\circ} 03.913^{\prime}$. On 
garden and on roadside trees. Among lagunes, on the place of former mangrove and coastal forest vegetation. 20. Aug. 2003.

03260: Southern coast of VITI LEVU Island ('Coral Coast') in the high rainfall area. Between NAVULA and LOMBAU villages, along the Queens Road, at 2-3 m alt. S $18^{\circ} 12.483^{\prime}, \mathrm{E} 178^{\circ} 10.813^{\prime} .10-12 \mathrm{~m}$ tall Pandanus tectorius dominated swamp forest adjoining mangroves along lagunes on the coastal plain. 20. Aug. 2003.

03261: Southern coast of VITI LEVU Island ('Coral Coast') in the high rainfall area. Near NABUKAVESI village, $10 \mathrm{~km} \mathrm{~N}$ of LOMBAU, at 30-60 m alt. S 1807-09', E $178^{\circ} 13.23-13.08^{\prime}$. Primary, wet lowland rainforest, on rugged volcanic hills, partly along streamlet. 20. Aug. 2003.

03262: North-eastern coast of VITI LEVU Island, at NATALEIRA eco-village. On the foothills of mountains behind the village, at 50-60 m alt. S 17³5.888', E $178^{\circ} 28.965^{\prime}$. Mesic rainforest on acidic, ferralitic soil. 21. Aug. 2003.

03263: North-eastern coast of VITI LEVU Island, near NAYARUTOKA village, $0-2 \mathrm{~m}$. S 17³3.339', E 178²4.746'. 10-12 m tall mangrove forest. 22. Aug. 2003.

03264: North-eastern coast of VITI LEVU Island, in the dry evergreen forest belt, SSE

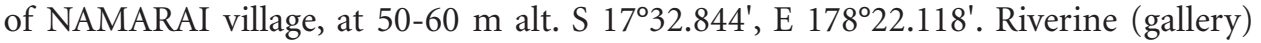
forest and shady volcanic tuff cliffs. 22. Aug. 2003.

03267: North coast of VITI LEVU Island. $15 \mathrm{~km}$ WSW of RAKIRAKI town, at 16-25 m alt. S $17^{\circ} 23.608^{\prime}, \mathrm{E} 178^{\circ} 15.408^{\prime}$. Dry semi-deciduous forest on volcanic breccia rocks. 22. Aug. 2003.

03269: Northern central part of VITI LEVU Island. SW ridge of Mt TAMANIVI (Mt Victoria), at $800 \mathrm{~m}$ alt, $3 \mathrm{~km} \mathrm{~S}$ of NAVAI, $28 \mathrm{~km}$ on the road from Tavua. S 17040.410', E 17759.363'. Mossy montane rainforest (cloud forest). 23. Aug. 2003.

03271: Central VITI LEVU, below the NE escarpment of RAIRAIMATUKU Plateau, $1 \mathrm{~km} \mathrm{NW}$ of NAQELEWAI village, in the NABOUBUCO River Valley, at $250 \mathrm{~m}$ alt. S $17^{\circ} 42.650^{\prime}, \mathrm{E} 178^{\circ} 04.950^{\prime}$. Wet lowland rainforest rich in epiphylls. 24. Aug. 2003.

03273: Central VITI LEVU, NE edge of RAIRAIMATUKU Plateau, above NAQELEWAI village, S from 'Barclay's Point', at 855-945 m alt. S 17²44.208-264', E 17803.300-573'. Montane mossy (cloud) forest. 24. Aug. 2003.

03274: Central VITI LEVU, on the ridge of RAIRAIMATUKU Plateau $10 \mathrm{~km}$ SSE of NAVAI. S $17^{\circ} 43.503^{\prime}$, E $178^{\circ} 02.252^{\prime}$. Mossy elfin forest (cloud forest) built up mostly by Cyathea sp. and by the 3-4 m tall Alpinia boia, at 990-1010 m alt. 24. Aug. 2003.

03275: Central VITI LEVU, at the N end of RAIRAIMATUKU Plateau, along the road,

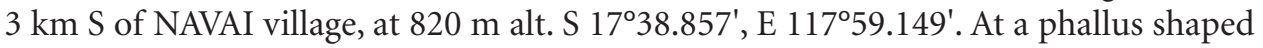
volcanic conglomerate rock, surrounded my mesic rainforest. 24. Aug. 2003.

03276: SE part of VITI LEVU Island. COLO-I-SUVA $10 \mathrm{~km} \mathrm{NNE} \mathrm{of} \mathrm{SUVA} \mathrm{town,} \mathrm{in}$ the garden or Raintree Lodge, at $185 \mathrm{~m}$ alt. S $18^{\circ} 03.498^{\prime}$, E $178^{\circ} 27.44^{\prime}$. Secondary wet lowland rainforest (by $4240 \mathrm{~mm}$ annual rainfall). 25. Aug. 2003.

03278: Central part of TAVEUNI Island. DES VOEUX Peak. S 1650.638', W 17957.991'. $1.5-2.5 \mathrm{~m}$ tall bush formed by a pachycaul Asteraceae on the summit ridge, at 11801194 m alt. 27. Aug. 2003. 
03279: Central part of TAVEUNI Island. NE side of DES VOEUX Peak, at 1040-1150 $\mathrm{m}$ alt. S $16^{\circ} 50.295-445^{\prime}, \mathrm{W} 179^{\circ} 58.037-224^{\prime} .4-8 \mathrm{~m}$ tall mossy elfin forest, very rich in epiphytes. 27. Aug. 2003.

03280: Central part of TAVEUNI Island. NE side of DES VOEUX Peak, at $960 \mathrm{~m}$ alt. S 16050.220, W 17958.369'. 10-20 m tall, mossy montane rainforest. 27. Aug. 2003.

03281: NE part of TAVEUNI Island. On the ridge between QELENI and WELAGI villages, at $500 \mathrm{~m}$ alt. S $16^{\circ} 45.285^{\prime}, \mathrm{W} 179^{\circ} 54.306^{\prime}$. Submontane rainforest with many tree-ferns along brook. 27. Aug. 2003.

03282: E side of TAVEUNI Island. Around the $I^{\text {st }}$ TAVORO Waterfalls, above KOROVOU (BOUMA) village, at 10-100 m alt. S 1649.608-620', W 17952.555-850'. Very wet lowland rainforest and woody cultivation, with many tree-fens and epiphytes. 29. Aug. 2003.

03283: LAVENA Beach along the SE coast of TAVEUNI Island at $1-3 \mathrm{~m}$ alt. S $16^{\circ} 52.261-$ 491', W 179 52.162-755'. Coconut growth intermixed with the remnants of coastal bush and forest, formed by Barringtonia asiatica, Pandanus and Hibiscus tiliaceus. 30. Aug. 2003.

03284: NABOUCANDRA Beach on the SE coast of TAVEUNI Island, W of LAVENA village. Degraded Barringtonia - Hibiscus coastal forest intercropped by coconut (Cocos nucifera), at 2-4 m alt. S 1652.303', W 17953.574'. 30. Aug. 2003.

03285: WAINISARI Beach on the SE coast of TAVEUNI Island, between NABA village and the estuary of WAINIBAU Stream, at 4-10 $\mathrm{m}$ alt. S $16^{\circ} 52.322-386^{\prime}$, W $179^{\circ} 53.270$ 864 '. Rocky (volcanic) coastal forest with many tree-ferns, here and there intercropped by coconut (Cocos nucifera). 30. Aug. 2003.

03286: WAINIBAU Stream valley on the SE coast of TAVEUNI Island, below the gorge of WAINIBAU Waterfalls, at 1-15 m altitude. S 16 51.950'-52.386', W 17954.270-331'. Lowland rainforest. 30. Aug. 2003.

03287: NEISOGO (NASELESELE) Point on the NE end of TAVEUNI Island opposite of the small VIUBANI Island, at 0.5-1.5 m altitude, S 16²41.964', W 179 $51.895^{\prime}$. Coconut growth intermixed with coastal Barringtonia - Hibiscus tiliaceus bushes. 31. Aug. 2003.

03288: Central part of TAVEUNI Island. Along the road from WAIRIKI to DES VOEUX Peak, on the NW slopes at 600-700 m alt. S 1649.639-758', W 17958.826-943'. Montane rainforest rich in epiphytes. 1. Sept. 2003.

03289: Central part of TAVEUNI Island. Along the road from WAIRIKI to DES VOEUX Peak, on the NW slopes, at 715-750 m alt. S 16²49.831-915', W 17958.643-731'. Mossy montane rain forest with transitions to elfin forest. 1. Sept. 2003.

03290: NW coast in the SW part of TAVEUNI Island. Taveuni Estates above SAQULU, on the foothills of Mt ULUIGALAU, at $360 \mathrm{~m}$ alt. S 16²51.295', E 17959.024'. Degraded submontane rainforests. 3. Sept. 2003.

03291: SW end of TAVEUNI Island. Between Vuna Lagoon Lodge and VUNA village, at $0.5-3 \mathrm{~m}$ alt. S $16^{\circ} 58.141-212^{\prime}$, E 179 53.405-4223'. Coastal bush dominated by Barringtonia asiatica and Hibiscus tiliaceus, with coconut on volcanic rocks. 4 and 7 Sept. 2003. 
03292: S tip of TAVEUNI Island. NE slope of Mt DELAIVUNA, at 120-170 m alt. S $16^{\circ} 59.458^{\prime}$, E $179^{\circ} 55.201^{\prime}$. Somewhat disturbed, relatively dry lowland rainforest dominated by 20-30 m tall Barringtonia edulis. 4 Sept. 2003.

03293: $S$ tip of TAVEUNI Island. On the NE slopes and in the crater of the extinct TAVUYAGEA volcano, at 150-295 m altitude, S 1659.660-662', E 17955.296-360'. Relatively dry Barringtonia edulis dominated lowland rainforest on the outer slopes and wet rainforest with many epiphytes (incl. epiphylls) in the crater. 5. Sept. 2003.

03294: S tip of TAVEUNI Island, Behind the 'BLOW HOLE' at $8-10 \mathrm{~m}$ alt. S $17^{\circ} 00.260^{\prime}$, E $179^{\circ} 55.773$ '. Pandanus tectorius stand with transitions to coastal forest, on lava rocks. 6. Sept. 2003.

03295: SSE coast of TAVEUNI Island, WSW of NAVAKA-WAU, at $8 \mathrm{~m}$ alt. S $17^{\circ} 00.151^{\prime}$, E 179 55.926'. Barringtonia asiatica dominated coastal forest. 6 Sept. 2003.

03296: SW part of TAVEUNI Island, on the foothills at the SW end of the central mountain range, between SALIALEVU and NAQARAWALU villages, at 100-200 m alt. S 16 57.934-58.598', E 179 57.431-678'. Wet, degraded lowland rainforest and coconut plantations. 6 Sept. 2003.

03297: E side of TAVEUNI Island. Around the It TAVORO Waterfall, above KOROVOU (BOUMA) village, at 10-100 m alt. S 16²49.564-620', W 17952.420-800'. Cultivated area and degraded lowland rainforest. 8. Sept. 2003.

03298: E side of TAVEUNI Island. Around the II ${ }^{\text {nd }}$ TAVORO Waterfall, above KOROVOU (BOUMA) village, at 120-150 m alt. S 16²49.640-680', W 17952.960'-53.050'. Wet lowland rainforest, somewhat degraded. 8 Sept. 2003.

03299: E side of TAVEUNI Island. Between the II ${ }^{\text {nd }}$ and III ${ }^{\text {rd }}$ TAVORO Waterfalls, above KOROVOU (BOUMA) village, at 150-160 m alt. S 1649.564-620', W 17952.420-800'. Very humid primary lowland rainforest, rich in epiphytes and epiphylls. 8. Sept. 2003.

03300: Central part of KADAVU (Kandavu) Island. 2-3 km NNE of VUNISEA, along the Namara Road, on the slopes at 120-165 m alt. S 1901.731-880', E 178 $10.265-750^{\prime}$. Secondary lowland rainforest on lilac volcanic soil. 10. Sept. 2003.

03301: Central part of KADAVU (Kandavu) Island. 2-3 km NNE of VUNISEA, along the Namara Road, in a valley below waterfall, at 110-150 m alt. S 1901.826-923', E $178^{\circ} 775-815^{\prime}$. Less disturbed lowland rainforest along rocky streamlet. 10. Sept. 2003.

03302: West part of KADAVU (Kandavu) Island. $2.5 \mathrm{~km} \mathrm{~W}$ of VUNISEA, on the north coast, at 1-20 m altitude. S $19^{\circ} 02.705^{\prime}$, E $178^{\circ} 08.221^{\prime}$. Rocky, shady coastal forest dominated by Barringtonia asiatica, Ficus sp. and Hibiscus tiliaceus, with Entada liana on lava rocks and coastal bush with coconut and with Scaevola sp. on the coral sand of the beach. 11. Sept. 2003.

03303: Central part of KADAVU (Kandavu) Island. On the coastal ridge, $0.5-1.5 \mathrm{~km}$ N of VUNISEA, at 40-80 m altitude, on lilac volcanic soil. S 1902.390-529', E $178^{\circ} 09.554-608^{\prime}$. Dry evergreen microphyllous forest with emergent Allocasuarina trees, many creeping Flagellaria and with Schizaea dichotoma and Cyperaceae common in the ground layer. 12. Sept. 2003.

03304: Western part of KADAVU (Kandavu) Island. $2 \mathrm{~km}$ NE of TAVUKI village, at 170$200 \mathrm{~m}$ altitude, on orange-red volcanic soil. S 1903.829-04.102', E 17807.771-921'. 
Dry evergreen microphyllous forest with emergent Allocasuarina trees, many creeping Flagellaria and with Schizaea dichotoma and Cyperaceae common in the ground layer. 13. \& 15. Sept. 2003.

03305: Western part of KADAVU (Kandavu) Island. $3 \mathrm{~km}$ NNE of TAVUKI village, on a ridge at $220-240 \mathrm{~m}$ altitude, on orange-red volcanic soil. S $19^{\circ} 03.621-678^{\prime}, \mathrm{E}$ $178^{\circ} 07.743-844^{\prime}$. Dry evergreen microphyllous forest with emergent Allocasuarina trees, with transitions to mesic evergreen rain forest. 13. Sept. 2003.

03306: Western part of KADAVU (Kandavu) Island. $3.5 \mathrm{~km}$ NE of TAVUKI village, on the M slope and summit of KORONIQUALA Hill, at 250-370 m altitude. S 1903.298$366^{\prime}$, E $178^{\circ} 08.0 .25-126^{\prime}$. Mesic submontane rainforest with tree-ferns, no epiphylls. 13 Sept. 2003.

03307: Central part of KADAVU (Kandavu) Island. On the ridge SE of VUNISEA telecom tower at 100-120 m altitude, on lilac volcanic soil. S 1903.214-300', E $178^{\circ} 09.836-877^{\prime}$. Dry evergreen microphyllous forest with emergent Allocasuarina trees, many creeping Flagellaria and with Schizaea dichotoma and Cyperaceae common in the ground layer. 14. Sept. 2003.

03308: Central part of KADAVU (Kandavu) Island. In the first bay E of VUNISEA on the southern coast, at 1-4 m altitude. S 1902.940', E 178 $10.005^{\prime}$. Coastal bush and Barringtonia asiatica forest with transitions to dry evergreen microphyllous Allocasuarina forest. 14. Sept. 2003.

03309: Western part of KADAVU (Kandavu) Island. $2 \mathrm{~km} \mathrm{NE}$ of TAVUKI village, at 150-175 m altitude. On orange red volcanic soil. S 1904.097-101', E 178 07.980'08.249'. Dry evergreen microphyllous forest with emergent Allocasuarina trees, many creeping Flagellaria and with Schizaea dichotoma and Cyperaceae common in the ground layer. 15 Sept. 2003.

03310: Western part of KADAVU (Kandavu) Island. $3 \mathrm{~km}$ ENE of TAVUKI village, in a rocky streambed above waterfalls, at $120 \mathrm{~m}$ altitude. S $19^{\circ} 04.354^{\prime}, \mathrm{E} 178^{\circ} 08.700^{\prime}$. Streamside rainforest with tree-ferns and many buttressed trees, on big boulders. 15. Sept. 2003.

08012: TAVEUNI Island, DES VOEUX (DEVO) peak, near PWD Weather Station, on the summit ridge at 1180-1190 m alt. S 16050'27", W 17957'32-59.5".

Hab.: Elfin forest dominated by Cunonia sp. 11 Apr. 2008

08013: TAVEUNI Island, NW slope of DES VOEUX (DEVO) peak, below PWD Weather Station, at 900-1100 m alt. S 16 50'18-28", W 17957'57-58". Montane mossy forest. 11 Apr. 2008

08015: $\mathrm{N}$ end of TAVEUNI Island, coast near NAEANGAI village., at $1-2 \mathrm{~m}$ alt. $\mathrm{S}$ 16² $43^{\prime} 57^{\prime}$, W179 $51^{\prime} 22$. Mangrove like forest dominated by Heritiera litoralis. Apr. 2008

08020: Central VITI LEVU, on the ridge of RAIRAIMATUKU Plateau, $15 \mathrm{~km} \mathrm{~S}$ of MONASAVU, at $924-960 \mathrm{~m}$ alt. S $17^{\circ} 48^{\prime} 43^{\prime \prime}, \mathrm{E} 178^{\circ} 02^{\prime} 06^{\prime \prime}$. Elfin forest with transition to mossy montane forest. 15 Apr. 2008

08023: Central VITI LEVU, RAIRAIMATUKU Plateau, on a summit with telecommunication tower, $16 \mathrm{~km} \mathrm{~S}$ of MONASAVU, at $1265 \mathrm{~m}$ alt. S 17²47'31", E $178^{\circ} 01^{\prime} 13.9^{\prime \prime}$. Elfin forest. 15 Apr. 2008 
08028: Central VITI LEVU, on the ridge of RAIRAIMATUKU Plateau, $2 \mathrm{~km}$ E of MONASAVU Dam, on the SW side of a summit at 1050-1070 m alt. S 17²4'21", E $178^{\circ} 04^{\prime} 09^{\prime \prime}$. Shady, very wet elfin forest dominated by Dicksonia brackenridgei. 16 Apr. 2008

08029: Central VITI LEVU, RAIRAIMATUKU Plateau, NE side of a summit with telecommunication tower, $2 \mathrm{~km}$ E of MONASAVU Dam, at $1050 \mathrm{~m}$ alt. S 17²45'20", E 17804'11". Cyathea- Alpinia boia elfin forest. 17 Apr. 2008

08032: Northern part of VITI LEVU Island. NADARIVATU Nature Reserve SW of

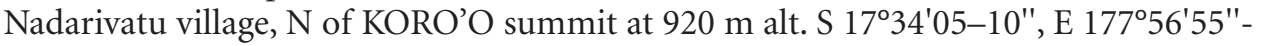
57'08". Mesic evergreen forest with Podocarpus neriifolius, Decussocarpus and Agathis vitiensis. 17 Apr. 2008

08033: Northern part of VITI LEVU Island. NAQUARANIBULUTI Nature Reserve $1 \mathrm{~km}$ E of NADARI-VATU village, along the track to Mt LOMALAGI, at $875 \mathrm{~m}$ alt. S $17^{\circ} 34^{\prime} 05^{\prime \prime}, \mathrm{E} 177^{\circ} 57^{\prime} 58^{\prime \prime}$. Mesic and dry evergreen forest with emergent Podocarpus neriifolius and Agathis vitiensis. 17. Apr. 2008

08034: Central VITI LEVU, RAIRAIMATUKU Plateau, near the FEA Station of MONASAVU Dam, along the road below the waterfalls, at $800 \mathrm{~m}$ alt., S 17²4 '38", E $178^{\circ} 03^{\prime} 15^{\prime \prime}$. On naturalised Piper trees. 18 Apr. 2008

08035: Central VITI LEVU, RAIRAIMATUKU Plateau, at the SE end of the artificial MONASAVU Lake, around the FEA Station, at 790-800 m alt., S 17²4'39.5", E $178^{\circ} 03^{\prime} 10^{\prime \prime}$. On planted Citrus trees and ornamental shrubs. 19 Apr. 2008

08036: SE end of VITI LEVU Island, COLO I SUVA Forest Res. $9 \mathrm{~km} \mathrm{~N}$ from SUVA capital town, $1 \mathrm{~km} \mathrm{~S}$ of Raintreee Lodge, at $200 \mathrm{~m}$ alt. S $18^{\circ} 03^{\prime} 47^{\prime \prime}$, E $178^{\circ} 27^{\prime} 24^{\prime \prime}$. Lowland rainforest at flat parts dominated by Pandanus. 20 Apr. 2008

08037: VITI LEVU Island, Veivatuloa Province, Coral Coast $7 \mathrm{~km} \mathrm{~N}$ of LOMBAU, in Nambukavesi Valley along Namosi Road, at $80 \mathrm{~m}$ alt. Epiphylls in lowland rain forest. 22 Apr. 2008

08038: SE part of VITI LEVU Island. COLO-I-SUVA $10 \mathrm{~km}$ NNE of SUVA town, in the garden of Raintree Lodge, at $185 \mathrm{~m}$ alt. S $18^{\circ} 03.498^{\prime}$, E $178^{\circ} 27.44^{\prime}$. On dead leaf fallen from canopy. 20. Apr. 2008. 